\title{
GRP78 clustering at the cell surface of neurons transduces the action of exogenous alpha-synuclein
}

\author{
S Bellani ${ }^{1}$, A Mescola ${ }^{2}$, G Ronzitti ${ }^{3,4}$, H Tsushima $^{3}$, S Tilve ${ }^{3}$, C Canale ${ }^{2}$, F Valtorta*, ${ }^{*, 1,5}$ and E Chieregatti ${ }^{*, 3,5}$
}

Mutation or multiplication of the alpha-synuclein (Syn)-encoding gene is frequent cause of early onset Parkinson's disease (PD). Recent evidences point to the pathogenic role of excess Syn also in sporadic PD. Syn is a cytosolic protein, which has been shown to be released from neurons. Here we provide evidence that extracellular Syn induces an increase in surface-exposed glucose-related protein of $78 \mathrm{kDa}$ (GRP78), which becomes clustered in microdomains of the neuronal plasma membrane. Upon interacting with Syn, GRP78 activates a signaling cascade leading to cofilin 1 inactivation and stabilization of microfilaments, thus affecting morphology and dynamics of actin cytoskeleton in cultured neurons. Downregulation of GRP78 abolishes the activity of exogenous Syn, indicating that it is the primary target of Syn. Inactivation of cofilin 1 and stabilization of actin cytoskeleton are present also in fibroblasts derived from genetic PD patients, which show a dramatic increase in stress fibers. Similar changes are displayed by control cells incubated with the medium of PD fibroblasts, only when Syn is present. The accumulation of Syn in the extracellular milieu, its interaction with the plasma membrane and Syn-driven clustering of GRP78 appear, therefore, responsible for the dysregulation of actin turnover, leading to early deficits in synaptic function that precede neurodegeneration.

Cell Death and Differentiation (2014) 21, 1971-1983; doi:10.1038/cdd.2014.111; published online 15 August 2014

Alpha-synuclein (Syn), a small soluble protein abundant in the brain, ${ }^{1}$ is implicated in the pathogenesis of various neurodegenerative disorders, among them Parkinson's disease (PD). ${ }^{2}$ Syn is the major component of intraneuronal inclusions found in the brain of PD patients. ${ }^{3}$ Distinct mutations ${ }^{4}$ as well as multiplications of the Syn-encoding gene $(S N C A)^{5-7}$ have been identified in families with genetic forms of PD. Moreover, genome-wide association studies correlated polymorphisms in regions of the SNCA gene to sporadic cases of PD. ${ }^{8}$

Syn is a natively unfolded protein enriched in presynaptic terminals, where it dynamically associates with synaptic vesicle membrane and lipid rafts. ${ }^{9,10} \mathrm{~A}$ plethora of possible Syn interactors has been recognized ${ }^{11}$ and among them cytoskeletal proteins. The influence of Syn on the microtubule-based cytoskeleton has been characterized in vitro. ${ }^{12}$ Recently, ${ }^{13}$ Syn has been shown to depolymerize actin cytoskeleton, a master regulator of synaptic function, ${ }^{14}$ raising the possibility that the effects of Syn on synaptic vesicle dynamics are mediated by its interaction with actin.

Syn is localized in the cytosolic compartment, is released from cultured neurons and is present in biological fluids ${ }^{15}$ indicating that Syn may also act extracellularly, participating in cell-to-cell transmission of pathology. Intraneuronal inclusions were found in healthy engrafted cells decades after transplantation in PD patients, and the host-to-graft transmission of Syn pre-formed fibrils has been demonstrated to occur in the mouse brain. ${ }^{16}$

Here we show that exposure of neurons to extracellularly delivered wild-type (wt) or A30P-mutant monomeric Syn leads to cofilin 1 inactivation with the ensuing stabilization of microfilaments. These effects depend on Syn-driven clustering at the cell surface of the endoplasmic reticulum (ER) protein glucose-related protein of $78 \mathrm{kDa}$ (GRP78). We propose actin cytoskeleton modification as the initial step in Syn-induced neurodegeneration, thereby actin and actinbinding proteins might constitute an effective target for screening and therapy of PD.

\section{Results}

Extracellular Syns stabilize the actin cytoskeleton. We previously demonstrated that Syn can physiologically control actin turnover, activity that is altered in the Syn A30P mutant. ${ }^{13}$ As Syn is released from neurons, we wondered whether high levels of extracellular Syn could affect actin dynamics in a similar way. The morphology of the actin cytoskeleton was monitored in young hippocampal neurons

\footnotetext{
${ }^{1}$ Division of Neuroscience, San Raffaele Scientific Institute and Vita-Salute University, Milan 20132, Italy; ${ }^{2}$ Department of Nanophysics and Brain Technologies, Istituto Italiano di Tecnologia, Genoa 16163, Italy and ${ }^{3}$ Department of Neuroscience and Brain Technologies, Istituto Italiano di Tecnologia, Genoa 16163, Italy

${ }^{*}$ Corresponding authors: E Chieregatti, Department of Neuroscience and Brain Technologies, Istituto Italiano di Tecnologia, Via Morego 30, Genoa 16163, Italy. Tel: +3901171781740; Fax: +3901171781230; E-mail: evelina.chieregatti @iit.it

or F Valtorta, DIBIT 3A2, San Raffaele Scientific Institute, Via Olgettina 58, Milan 20132, Italy. Tel: +390226434826; Fax: +390226434844; E-mail: valtorta.flavia @ hsr.it ${ }^{4}$ Current address: Genethon, Evry, 91000 France.

${ }^{5}$ These authors shared last authorship.

Abbreviations: Syn, alpha-synuclein; PD, Parkinson's disease; SNCA, human Syn-encoding gene; ER, endoplasmic reticulum; GRP78, glucose-related protein of $78 \mathrm{kDa}$; wt, wild-type; FPLC, fast protein liquid chromatography; LatA, latrunculin A; DIV, day(s) in vitro; GFP, green fluorescent protein; UPR, unfolded protein response; elF2 $\alpha$, eukaryotic translation initiation factor 2 subunit alpha; IP, immunoprecipitation; AFM, atomic force microscopy; DTSSP, 3,3'-dithiobis[sulfosuccinimidylpropionate]; siRNA, short interfering RNA

Received 05.1.14; revised 02.6.14; accepted 01.7.14; Edited by N Bazan; published online 15.8.14
} 
after incubation for $1 \mathrm{~h}$ with $1 \mu \mathrm{M}$ purified human recombinant wt or A30P Syn. The recombinant proteins were soluble and monomeric, as shown by fast protein liquid chromatography (FPLC) and Coomassie staining (Supplementary Figures 1A and B). As compared with control samples, neurons exposed to extracellular Syns were enriched in actin structures (Figure 1a). The subtraction of $\beta$-tubulin III immunofluorescent areas from fluorescent phalloidin-stained areas revealed the actin protrusion areas (Supplementary Figure 2), which were significantly increased in Syn-treated neurons (Figure 1b). Syn-driven increase in actin structures was even more evident upon exposure to latrunculin A (LatA), ${ }^{17}$ which, by sequestering actin monomers, induced almost complete depolymerization of actin in control neurons, whereas, in neurons incubated with Syns, spots of polymerized actin were scattered throughout the cell body and along the neurites. The effect of Syns was dose-dependent and the dose-response curve appeared left-shifted for the A30P mutant; the increased resistance of actin to depolymerization became significant after exposure to $0.1 \mu \mathrm{M}$ A30P Syn and to $1 \mu \mathrm{M}$ wt Syn (Figure 1c). Microfilament stability was evaluated in mature neurons, in order to determine whether the effect of Syns was appreciable at later stages of development. As in young neurons, the exposure of 14 day(s) in vitro (DIV) neurons to extracellular wt or A30P Syn determined a significant increase in actin protrusion areas, which became more evident after LatA treatment (Figures 1d and e).

The slowing in actin turnover induced by Syns might have functional consequences, such as defects in axon elongation in developing neurons. To address this possibility, 3 DIV neurons were exposed for $1 \mathrm{~h}$ to $1 \mu \mathrm{M}$ Syns. The axons of neurons exposed to Syns showed an average 10\% decrease in length (Figure 1f). Unfolded Syns and Syns released from neurons affect
actin dynamics as the purified protein. In mouse brain,
Syn is physiologically present in both monomeric and
tetrameric form. ${ }^{18,19}$ To confirm that the observed
activity of Syns on the cytoskeleton is due to the unfolded
protein, purified Syns were denatured by boiling for 5 min,
and were added to cultured neurons as above. Denatured
and non denatured Syns affected the morphology of actin
cytoskeleton in a similar way, inducing both a significant
increase in actin protrusion areas and resistance to
depolymerization (Figures 2 a and b). To exclude
the possibility that the effect of Syn was due to fibril
formation, we used three distinct Syn peptides: P34 (aa
$34-45$ ), that is, the glycosphingolipid-binding domain of Syn,
P61 (aa $61-78$ ), that is, the cholesterol-binding domain of
Syn that has been shown to aggregate ${ }^{20,21}$ and P12 (aa $12-$
23), a peptide with no recognized function. None of these
peptides determined measurable effects on cytoskeleton
stabilization, suggesting that the whole protein, monomeric
and unfolded, is required for its action on microfilaments
(Figures $2 a$ and b).
To evaluate whether Syns, released from neurons, could
induce effects similar to the recombinant protein, we collected
the culture media of neurons electroporated with constructs
coding for wt Syn, A30P Syn or for green fluorescent protein and Differentiation
(GFP) as control. Western blot analysis confirmed the presence of the exogenous proteins in cell homogenates as well as in the $48 \mathrm{~h}$-conditioned media (Figures $2 \mathrm{c}$ and d). After high-speed centrifugation of the media as in, ${ }^{22}$ the bulk of wt and A30P Syn was detectable in the supernatant (S100), and only trace amounts (15\%) were present in the pellet (P100), where the exosomal marker flotillin 1 was retrieved (Figure 2e).

The $\mathrm{S} 100$ fraction of the medium isolated from GFP- or Syn-electroporated neurons was added to developing neurons in culture. Similarly to recombinant Syns (Figure 1a), Syn-containing media induced stabilization of the actin network, with increased resistance to LatA-induced depolymerization and increase in actin protrusion areas (Figures $2 \mathrm{f}$ and $\mathrm{g}$ ).

These results indicate that the presence of high dosage of extracellular Syns, either released from overexpressing neurons or added as recombinant proteins to the culture medium, modifies actin cytoskeleton structure. Neurons show increased number of lamellipodia and filopodia and increased resistance to depolymerization, with A30P Syn being more potent than wt Syn in inducing these effects.

Extracellular Syns activate an intracellular signaling cascade leading to cofilin 1 inactivation. We investigated the signal transduction pathway(s) responsible for the actin stabilizing activity of extracellular Syns. Exposure of 14 DIV hippocampal neurons to $1 \mu \mathrm{M}$ extracellular wt and A30P Syn for $1 \mathrm{~h}$ determined a two- and threefold increase, respectively, in the fraction of active GTP-bound Rac 1, recovered from neuronal lysates by pull-down assays (Figures 3a and b). Syns activate Rac 1 at $\sim 15-20 \%$ of the maximal value induced by GTP $\gamma \mathrm{S}$. Immunoblotting revealed that extracellular wt and A30P Syn determined a 1.5 and twofold increase, respectively, in the phosphorylation state of the Rac 1 effectors PAK 2 and cofilin 1 (Figures $3 c$ and d).

These results show that the formation of lamellipodia and membrane ruffles and the stabilization of microfilaments induced by Syns correlate with the activation of the effector proteins Rac 1, PAK 2, and cofilin 1, raising the possibility that these events have a causal role in determining the effect of Syns on cytoskeleton organization.

The changes in the dynamics of actin cytoskeleton represent early events responsible for functional deficits leading to degeneration of neurons. Therefore, we evaluated Syn-induced toxicity in neurons after chronic exposure to Syns by monitoring mitochondrial potential and cell mortality. At early time points, no difference could be detected between Syn-treated and untreated neurons. Only after exposure to recombinant Syns for 14 days, neurons exhibited mitochondrial damage and $30 \%$ of them showed membrane leakage due to cell death (Supplementary Figures $3 a-d$ ).

Extracellular Syns associate with GRP78 on the cell surface. To identify binding partners potentially mediating the action of extracellular Syns, homogenates from hippocampal neurons were separated by 2D SDS-polyacrylamide gel electrophoresis (PAGE). The few proteins recognized by purified wt or A30P Syn in an overlay assay (Figure 4a) were analyzed by MALDI-TOF. Among the proteins 

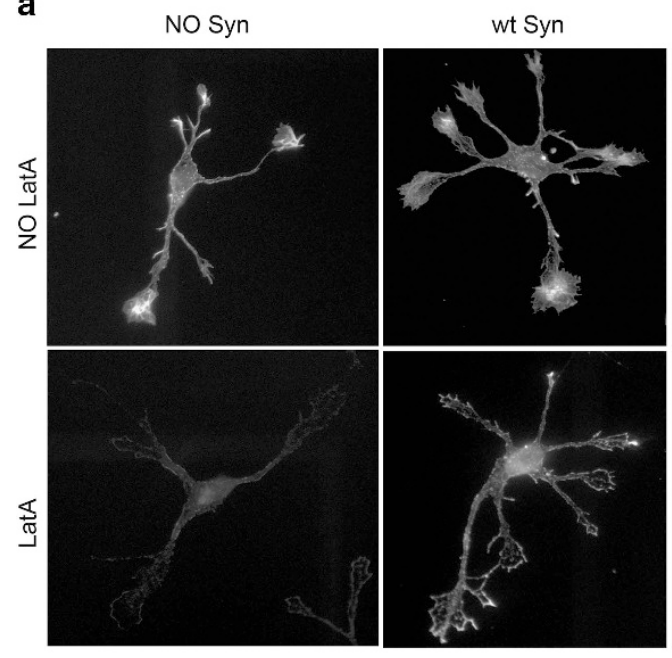

d

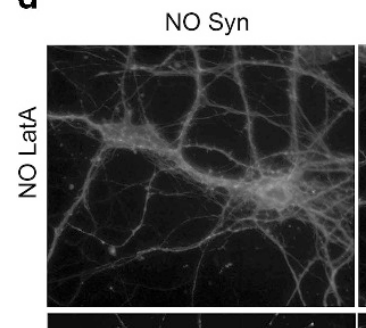
NO Syn
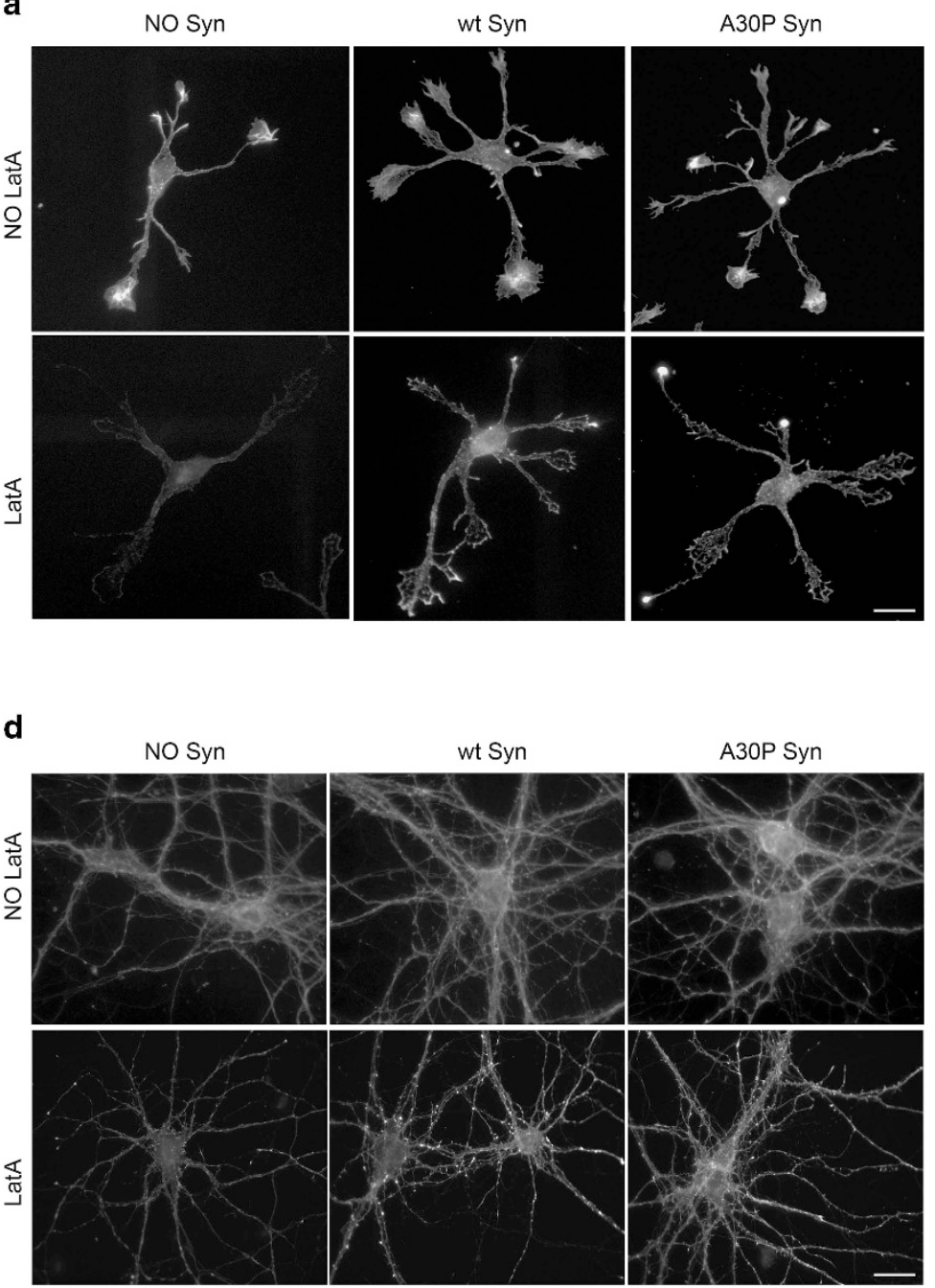

wt Syn
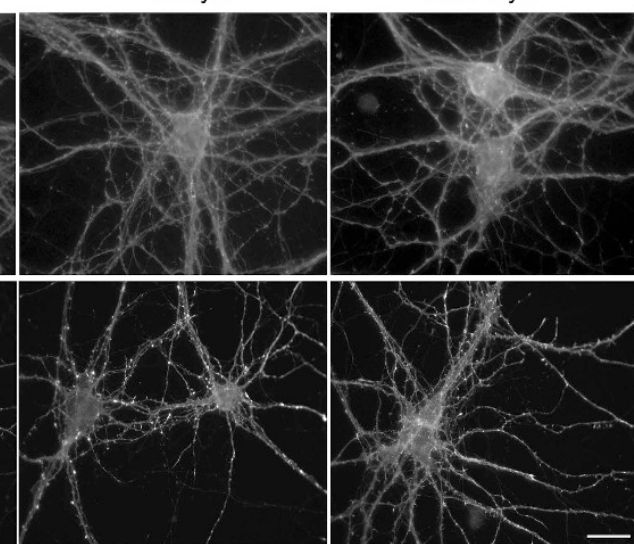

b
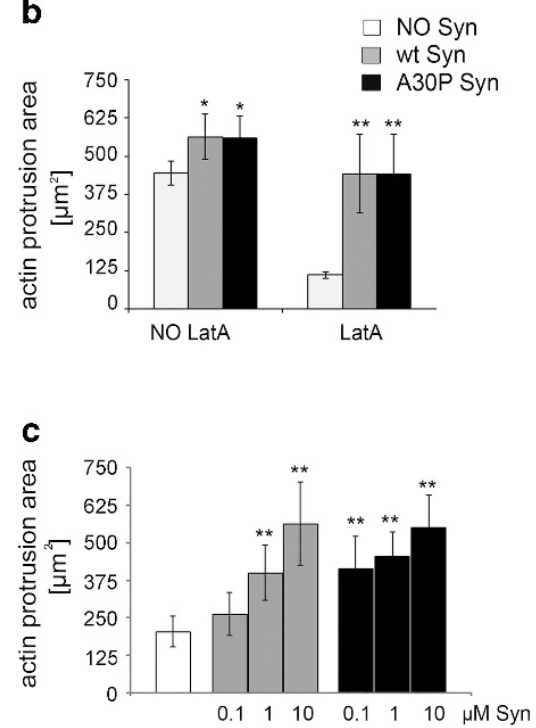

e
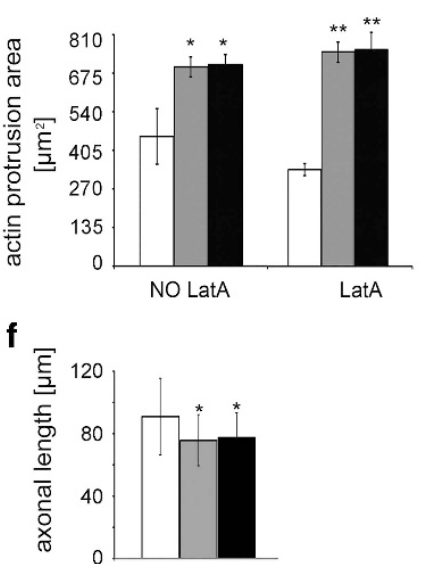

Figure 1 Extracellular wt and A30P Syn affect actin cytoskeleton. (a) F-actin distribution, as revealed by fluorescent phalloidin staining, in 1 DIV embryonic hippocampal neurons incubated in the absence or presence of $1 \mu \mathrm{M}$ purified wt or A30P Syn for $1 \mathrm{~h}$ and examined either under control conditions (NO LatA), or after a $1 \mathrm{~h}$ incubation with $1 \mu \mathrm{M}$ LatA (LatA). (b) Quantitative evaluation of actin protrusion areas in neurons treated as in $\mathbf{a}$. Actin protrusion areas were calculated by subtracting projection areas of $\beta$-tubulin III immunofluorescence from F-actin projection areas. (c) Dose-dependent effects of extracellular Syns on actin protrusion areas. Neurons were treated for $1 \mathrm{~h}$ with increasing concentrations of purified wt or A30P Syn, and then exposed to LatA for an additional hour (NO Syn: neurons not treated with Syn). Actin protrusion areas were calculated as in b. (d) F-actin distribution in 14 DIV embryonic hippocampal neurons incubated in the absence or presence of $1 \mu \mathrm{M}$ purified wt or A30P Syn for $1 \mathrm{~h}$; neurons were treated and stained as in a. (e) Quantitative evaluation of actin protrusion areas of neurons treated as in a, calculated as in $\mathbf{b}$. (f) Quantitative evaluation of average axonal length measured by Sholl analysis of 3 DIV embryonic hippocampal neurons incubated with or without $1 \mu \mathrm{M}$ purified wt or A30P Syn. In $\mathbf{b}$, $\mathbf{c}$ and $\mathbf{e}$, data are expressed as mean values \pm S.D.; $n=60$ neurons from three independent cultures. In $\mathbf{f}$, data are expressed as mean values \pm S.D.; $n=100$ neurons from three independent cultures. Statistical significance was determined by one-way ANOVA followed by Dunnett's test for multiple comparison, ${ }^{*} P<0.05$; ${ }^{\star *} P<0.01$. Bar in a and $\mathbf{d}$ : $20 \mu$ m

detected, we focused on GRP78, an ER chaperone detectable at the cell surface of different cell types, where it acts as a binding protein for various ligands. ${ }^{23}$ To test the hypothesis that GRP78 may be enriched at the plasma membrane upon incubation with Syns, hippocampal neurons, incubated with $1 \mu \mathrm{M}$ Syns for 1 or $4 \mathrm{~h}$, were labeled with a membrane-impermeable biotin-conjugated compound and cell-surface proteins were pulled down. Immunoblot analysis (Figures $4 \mathrm{~b}$ and $\mathrm{c}$ ) showed a $\sim$ threefold increase in surface-exposed GRP78 in neurons treated with extracellular Syns. Syns also appeared biotin-labeled, suggesting that extracellularly-delivered Syns bind to the cell surface of neurons.
GRP78 is known to translocate the plasma membrane in conditions of cellular stress. ${ }^{23}$ However, GRP94, GRP78, phosphorylated eukaryotic translation initiation factor 2 subunit alpha (elF2 $\alpha$ ) and activated caspase 3 levels were indistinguishable from controls (Figure 4d), indicating no activation of the unfolded protein response (UPR) or increased apoptosis after $4 \mathrm{~h}$ of Syn treatment.

The localization of both GRP78 and Syns at the plasma membrane was suggestive for their reciprocal interaction at the cell surface. Immunoprecipitation (IP) experiments performed in neuroblastoma N2A cells exposed to wt or A30P Syn in the presence of the membrane impermeable cross-linker 3,3'-dithiobis[sulfosuccinimidylpropionate] showed that Syns 
a

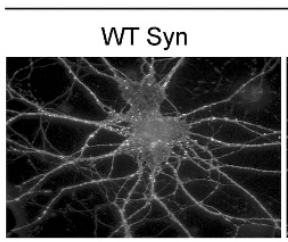

NO Syn
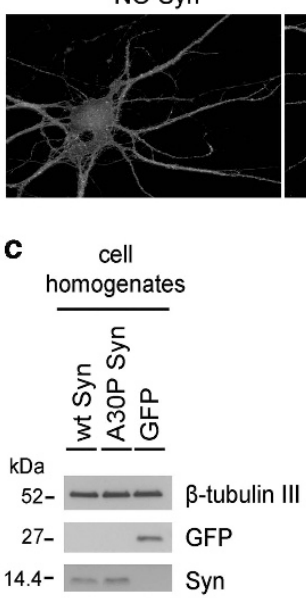

f

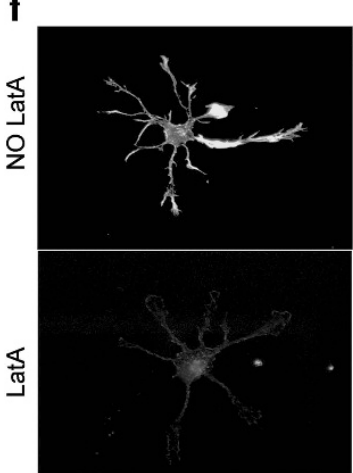

LatA

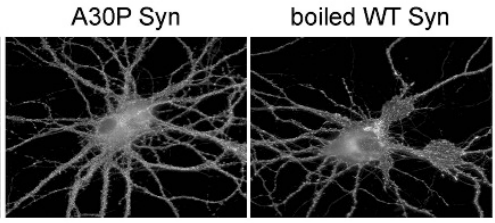

P12

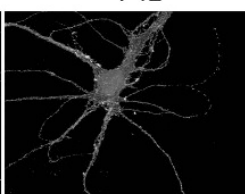

d
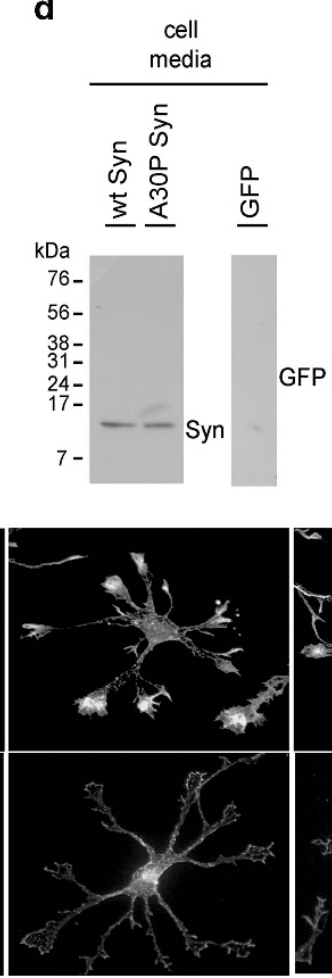

P34

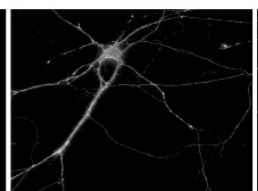

boiled A30P Syn

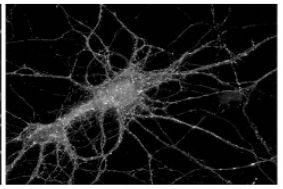

P61

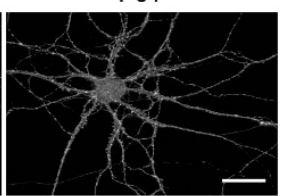

b

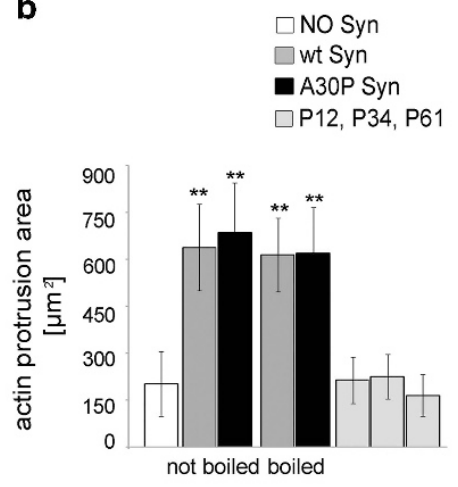

e

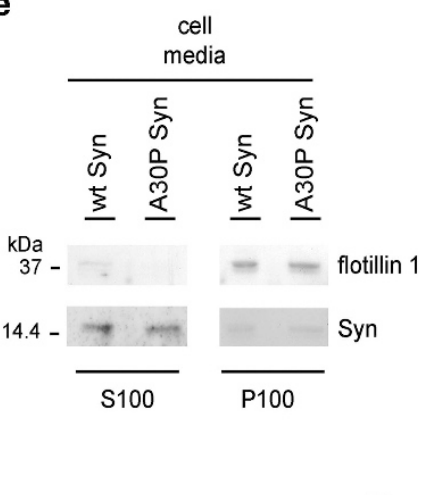

g

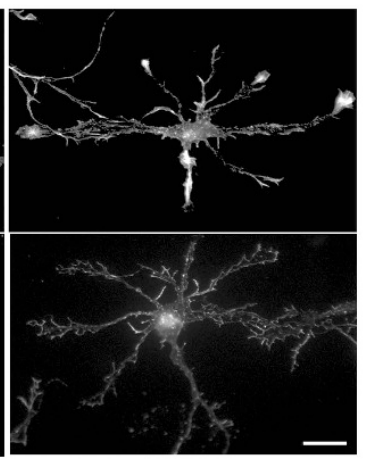

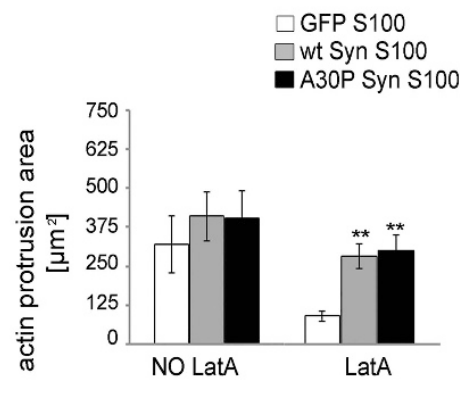

Figure 2 Unfolded and released wt and A30P Syn affect actin cytoskeleton similarly to the purified protein. (a) F-actin distribution of 14 DIV embryonic hippocampal neurons incubated without or with $1 \mu \mathrm{M}$ purified wt or A30P Syn, either untreated or boiled; or with $1 \mu \mathrm{M}$ Syn synthetic peptides (P12, P34, P61). Neurons were treated with LatA and stained as in Figure 1a. (b) Quantitative evaluation of actin protrusion areas of neurons treated as in a, calculated as in Figure 1b. (c) Immunoblots of cell homogenates from 2 DIV hippocampal neurons electroporated with coding constructs for either green fluorescent protein (GFP) or wt or A30P Syn. $\beta$-Tubulin III is shown as an internal standard. (d, e) Immunoblots showing the presence of Syn in the culture media collected from 2 DIV hippocampal neurons electroporated as in c (d), and in their soluble and vesicular fractions (e) separated by ultracentrifugation (S100 and P100, respectively). Flotillin 1 is shown as a marker of exosomal membranes. (f) F-actin distribution in 1 DIV hippocampal neurons incubated ON with S100 fractions from GFP-, wt Syn- or A30P Syn-expressing neurons; neurons were treated and stained as in Figure 1a. (g) Quantitative evaluation of actin protrusion areas of neurons treated as in $\mathbf{f}$, calculated as in Figure $1 \mathbf{b}$. In $\mathbf{b}$ and $\mathbf{g}$, data are expressed as mean values \pm S.D.; in b, $n=30$ neurons from two independent cultures, in $\mathbf{g}, n=60$ neurons from three independent cultures. Statistical significance was determined by one-way ANOVA followed by Dunnett's test for multiple comparison, ${ }^{* *} P<0.01$. Bar in a and f: $20 \mu \mathrm{m}$

are co-immunoprecipitated with GRP78, indicating their presence in a complex at the cell surface (Figure 4e). Consistent results were obtained in neurons biotin-labeled after incubation with Syns: the anti-GRP78 antibody was able to co-immunoprecipitate surface-bound biotin-labeled Syns (Figure 4f).

In the presence of extracellular Syns, surface-exposed GRP78 is increased and clustered in microdomains. The presence of GRP78 and Syn on the external leaflet of the plasma membrane was demonstrated in live, intact neurons by atomic force microscopy (AFM) experiments. Neurons were incubated in the presence or absence of Syn and subsequently exposed to anti-Syn or anti-GRP78 antibodies (Figure 5). The work required to detach the AFM functionalized probe, coated with a secondary antibody, from the primary antibody bound to specific antigens at the cell surface was evaluated. ${ }^{24,25}$ Distribution histograms (Figure 5a) and quantification of the distribution curves 
a

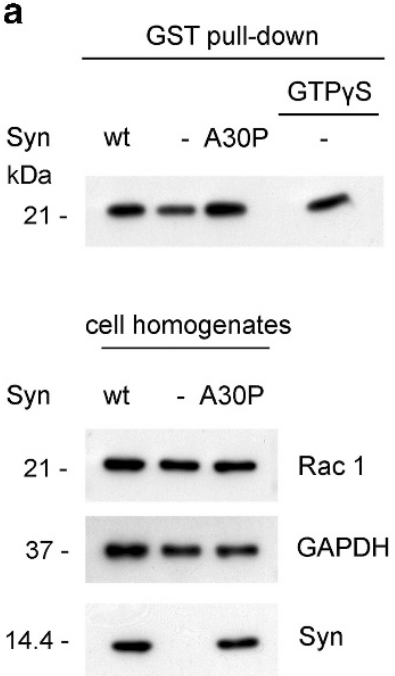

$$
\begin{aligned}
& \square \text { NO Syn } \\
& \text { wt Syn } \\
& \text { a3OP Syn }
\end{aligned}
$$

GTP-Rac 1

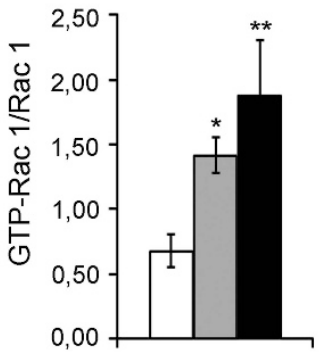

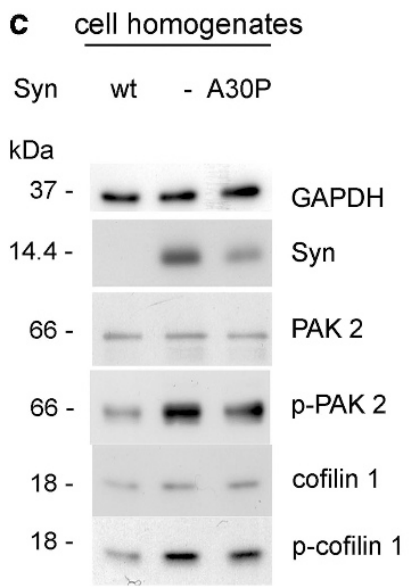

d

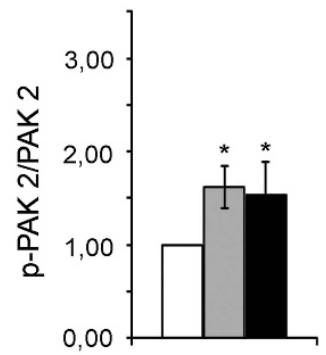

$\square$ NO Syn
$\square$ wt Syn
$\square$ A30P Syn

Figure 3 Intracellular pathways activated by extracellular Syns. (a) Upper panel: immunoblots showing Rac 1 activation in hippocampal neurons at 14 DIV, incubated for $1 \mathrm{~h}$ in the absence or presence of $1 \mu \mathrm{M}$ purified wt or A30P Syn. Activation of Rac 1 (GTP-Rac 1) was determined by GST pull-down assay. Neuronal lysate incubated with $0.1 \mathrm{mM}$ GTP $\gamma \mathrm{S}$ was used as a positive control for Rac 1 activation in the absence of Syn (this sample was diluted 1:10 with respect to GTP-Rac 1 pulled-down samples). Lower panel: immunoblots showing the total amount of Rac 1 in the cell homogenates used for the pull-down assay. The protein amount loaded corresponds to $10 \%$ of the starting samples used in the GST pull-down assay. (b) Quantification of the ratio between the GTP-Rac 1 and total Rac 1 bands analyzed by densitometry for each experimental condition shown in a. (c) Representative immunoblots showing levels of proteins indicated on the right in cell homogenates from hippocampal neurons treated in the absence or presence of purified wt or A30P Syn as in a. (d) Quantification of the ratios between the protein levels of p-PAK 2 and total PAK 2 (left panel) and p-cofilin 1 and total cofilin 1 (right panel) bands, analyzed by densitometry, for each experimental condition shown in c; values are normalized to those of control conditions (NO Syn). In a and c, GAPDH is shown as an internal standard. In $\mathbf{b}$ and $\mathbf{d}$, data are expressed as mean values \pm S.D.; $n=3$ independent experiments. Statistical significance was determined by one-way ANOVA followed by Dunnett's test for multiple comparison, ${ }^{*} P<0.05 ;{ }^{* \star} P<0.01$

(Figure $5 b$ ) show the increase in the normalized detachment work $\left(\mathrm{w} / \mathrm{w}_{0}\right)$ that indicates the presence of Syn at the cell surface in neurons exposed to Syn for $1 \mathrm{~h}$ and incubated with anti-Syn antibody. In neurons incubated with anti-GRP78 antibody after exposure to Syn, GRP78 presence at the cell surface was increased, as compared with controls. Thus, the results obtained with colloidal probes demonstrated the presence of Syn on the external leaflet of the cell membrane, as well as the increased presence of GRP78 after treatment with Syn.

The distribution of Syn and GRP78 on the plasma membrane was evaluated by using a sharp functionalized AFM tip, which has reduced area of contact with the sample. Force maps were acquired on the neuronal cell body. Molecular recognition was evaluated by measuring the number of rupture events (Figure $5 \mathrm{c}$ ) and the maximum rupture force $\left(F_{\max }\right)$ (Supplementary Figure 4$)$. After a $1 \mathrm{~h}$ incubation, Syn appeared localized at the cell surface of neurons in a non-homogeneous pattern, indicating the presence of preferential domains as binding sites. At the same time GRP78, almost absent in control conditions, was detected at the cell surface only after incubation of neurons with Syn and appeared enriched in specific microdomains of the membrane, similarly to Syn. Unspecific adhesion was evaluated in the presence of an excess of secondary antibody; in this case, the signals obtained with either antiSyn or anti-GRP78 antibody were below the recognition level (Figure $5 \mathrm{c}$ and Supplementary Figure 4).

Taken together, these results show that GRP78 is a potential binding partner for extracellular Syns in neurons 
through the formation of a complex that retains GRP78 molecules at the cell surface, and might act as a signaling platform for intracellular cascades.

GRP78 transduces Syn-driven stabilization of actin cytoskeleton. To determine whether GRP78 is required by Syns for triggering the activation of intracellular pathways, we downregulated GRP78 expression. Four days after electroporation with GRP78 targeted small interfering RNA (siRNAs), neurons exhibited a $\sim 60 \%$ decrease in GRP78 levels with respect to control neurons electroporated with scrambled siRNA (Supplementary Figures $5 \mathrm{~A}$ and $\mathrm{B}$ ), without evident cytoskeletal alterations (Supplementary Figure 5C). a

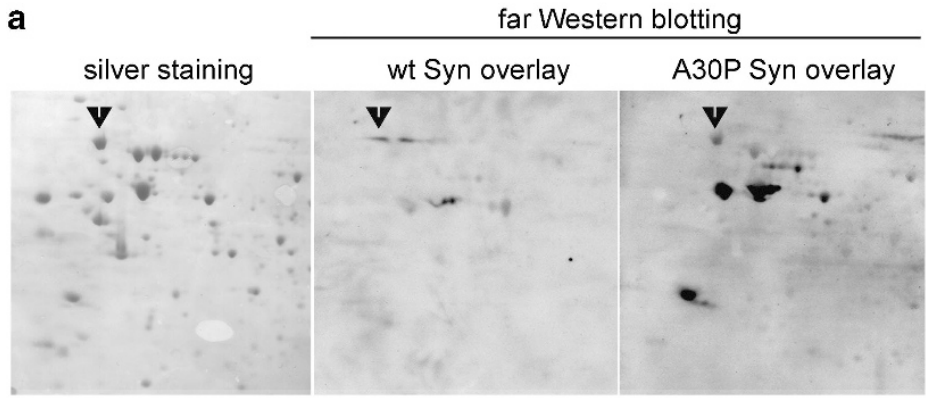

b

h $\frac{\text { cell homogenates }}{14}-\frac{\text { A30P Syn }}{14}$

$\mathrm{kDa}$

78 -

14.4 -

35 -

$5-$
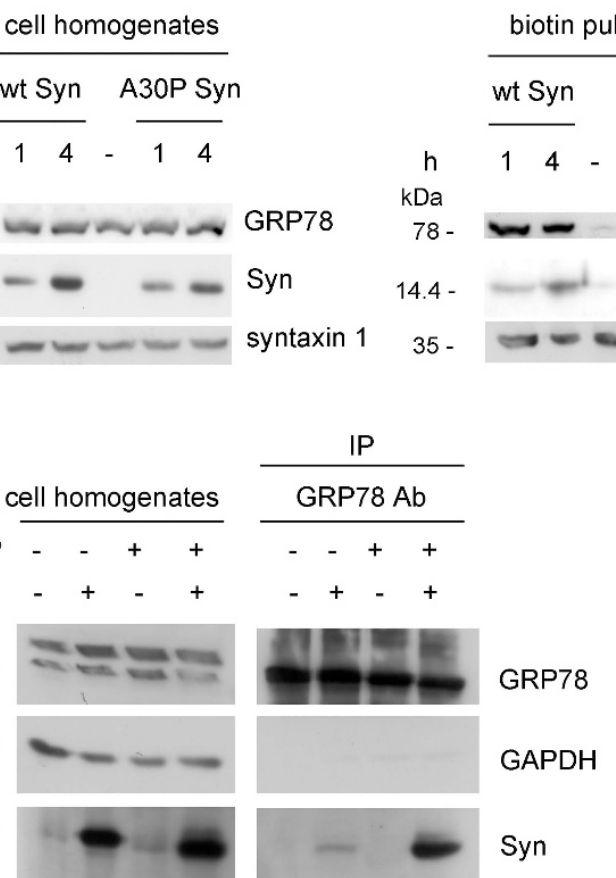

f

e
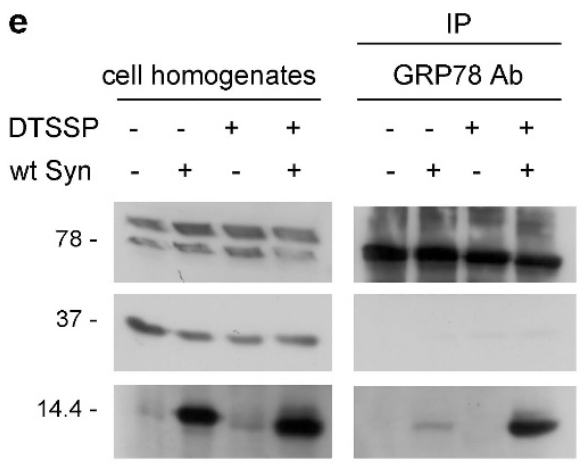

IP

cell homogenates GRP78 Ab unrelated $A b$

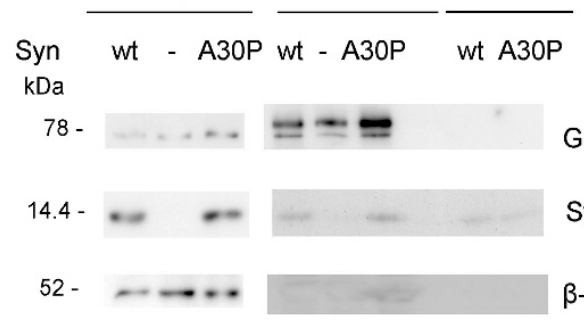

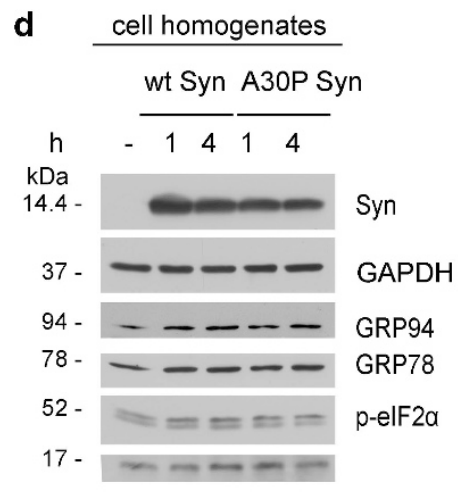

c

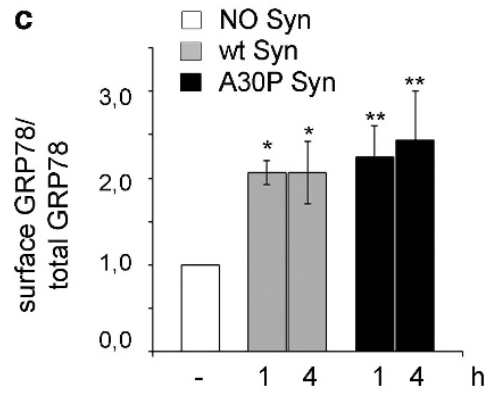

surface syntaxin 1

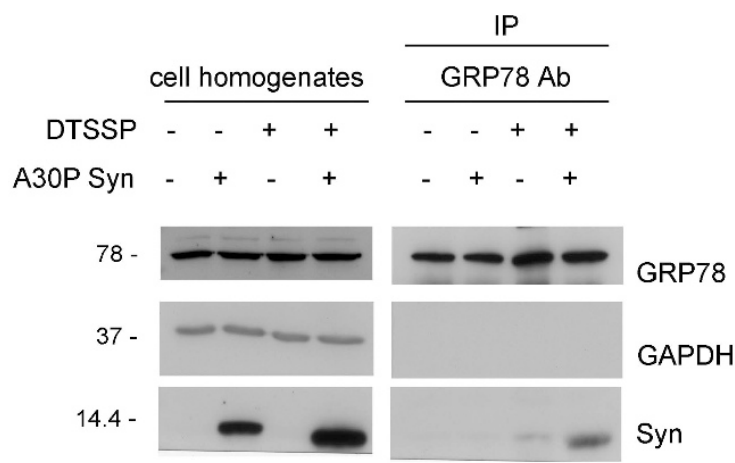

IP

GRP78 $A b$ unrelated $A b$

Syn wt - A30P wt A30P

$\mathrm{kDa}$

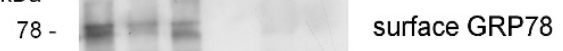

$14.4-$

surface Syn

52 -

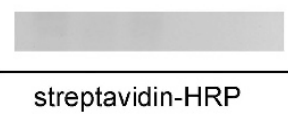


Consistent with the data shown in Figure 1, Syns induced an increased resistance to LatA-depolymerization in neurons electroporated with scrambled siRNA (Figures $6 a$ and $b$, CTRL SiRNA). Downregulation of GRP78 prevented the effects triggered by Syns, making actin resistance to depolymerization and areas of actin protrusions indistinguishable from those of control neurons (Figures $6 a$ and b, GRP78 siRNA). Consistently, in the absence of GRP78, Syn-driven inactivation/phosphorylation of cofilin 1 and phosphorylation of PAK2 were also abolished (Figures $6 \mathrm{c}$ and $\mathrm{d}$ ). Whereas a decrease in the expression levels of total cofilin 1 could be observed in neurons downregulated for GRP78, total PAK 2 levels remained unchanged.

These results show that GRP78 is required for the stabilizing action on the neuronal actin cytoskeleton triggered by extracellular Syns.
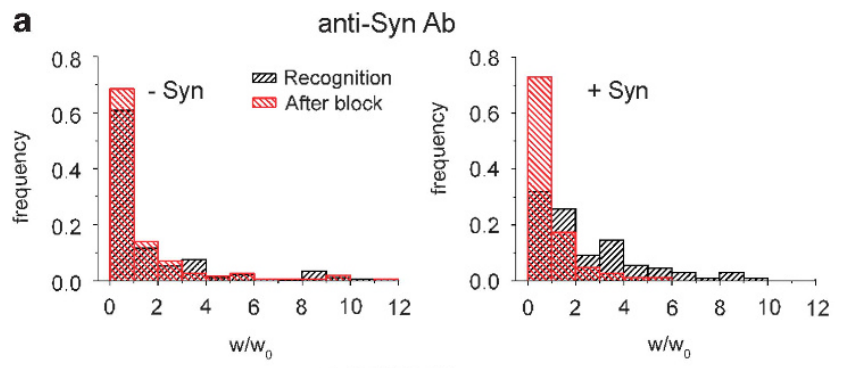

C Recognition
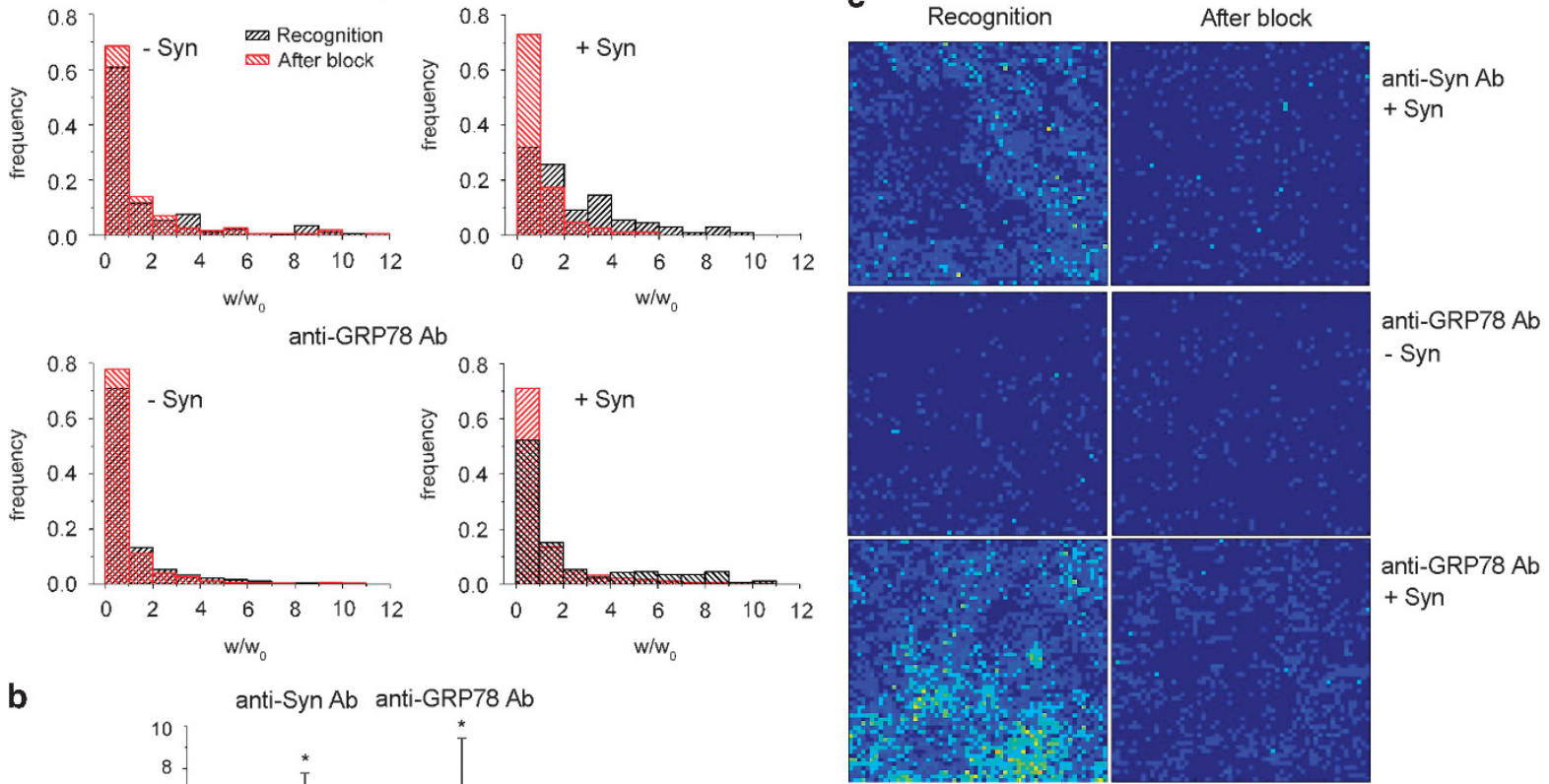

b

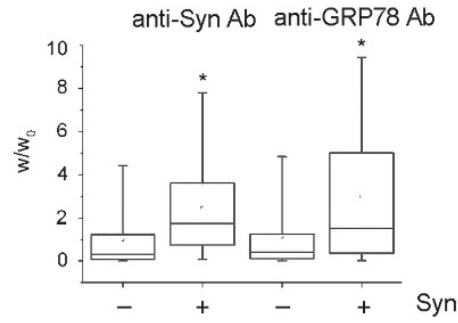

Figure 5 Clustering of Syn and GRP78 in microdomains on the external leaflet of the neuronal plasma membrane. (a) Distribution of the normalized detachment work $\left(\mathrm{w} / \mathrm{w}_{0}\right)$ in AFM assays (colloidal probe, $\varnothing 4 \mu \mathrm{m}$ ) performed in $10 \mathrm{DIV}$ cortical neurons in the absence (left graphs) or presence (right graphs) of $5 \mu \mathrm{M}$ wt Syn. Neurons were incubated with anti-Syn monoclonal antibody (upper graphs) or anti-GRP78 polyclonal antibody (lower graphs), followed by a rabbit anti-mouse or anti-goat secondary antibody, respectively (black). After the measurement, an excess of secondary antibody was delivered to the same sample to test for unspecific binding (red). (b) Quantification of $\mathrm{w} / \mathrm{w}_{0}$ in samples as in a, normalized for w/wo measured after block. (c) Pseudocolor bi-dimensional maps representing the number of recognition events obtained with a sharp functionalized AFM tip, during recognition in the presence of a species-specific secondary antibody (left panels) and after the block due to an excess of secondary antibody (right panels) (3600 force/distance curves, acquired on a $3 \times 3 \mu \mathrm{m}^{2}$ neuronal surface area). Maps were obtained in the presence or absence of $5 \mu \mathrm{M}$ wt Syn; in the figure, the specific binding of Syn (first row) and of GRP78 (third row) to the cell-surface of neurons is indicated by the increase in green-yellow pixels, clustered in discrete domains. In b, -Syn anti-Syn Ab, $n=174$; + Syn anti-Syn Ab, $n=198$; -Syn anti-GRP78 Ab, $n=269$; + Syn anti-GRP78 Ab, $n=301$. Statistical significance was determined by one-way ANOVA followed by Dunnett's test for multiple comparison, ${ }^{*} P<0.05$

Figure 4 Extracellular wt and A30P Syn increase the cell-surface localization of GRP78. (a) Parallel 2D gels of cell homogenates obtained from 14 DIV hippocampal neurons were either silver stained or transferred onto nitrocellulose membranes, overlaid with $1 \mu \mathrm{M}$ purified wt or A30P Syn and processed by far western blotting with anti-Syn antibody. The staining was revealed by ECL. Excised spots were analyzed by MALDI-TOF. The arrowhead indicates the spot identified as GRP78. (b) Immunoblots showing surface-bound proteins analyzed by biotinylated protein pull-down from 14 DIV hippocampal neurons, incubated for 1 or $4 \mathrm{~h}$ in the absence or presence of $1 \mu \mathrm{M}$ purified wt or A30P Syn and labeled with impermeable biotin. Biotinylated proteins, pulled down with streptavidin agarose beads, were analyzed by immunoblotting in parallel with their relative cell homogenates. Cell homogenates were loaded at $10 \%$ of the samples used in pull-down assays. (c) Quantification of the ratio between surface GRP78 (biotin pull-down) and total GRP78 (cell homogenates) bands analyzed by densitometry for each experimental condition in a. Values are normalized for control conditions (NO Syn). (d) Analysis of protein markers of UPR (GRP94, p-elF2 $\alpha$, GRP78) and apoptosis (activated caspase 3 ) in hippocampal neurons incubated in the presence or absence of purified wt or A30P Syn, as in b. GAPDH is shown as an internal standard. (e) Immunoblots showing co-IP of Syn and GRP78 in N2A cells, incubated for 20 min in the absence or presence of $1 \mu \mathrm{M}$ wt Syn (left panel) or A30P Syn (right panel) and of the impermeable cross-linker DTSSP and then precipitated with anti-GRP78 antibody. Cell homogenates are $5 \%$ of the samples used in IP assays. GAPDH is shown as an internal standard. (f) Immunoblots showing co-IP of Syn and GRP78 in hippocampal neurons incubated for $1 \mathrm{~h}$ in the absence or presence of $1 \mu \mathrm{M}$ purified wt or A30P Syn, surface-labeled with impermeable biotin and then precipitated with anti-GRP78 antibody (or with an unrelated antibody as a control). Immunoblots were analyzed for the total amount of GRP78, Syn and $\beta$-tubulin III (left panel) and for their surface retention, by streptavidinconjugated HRP (right panel). Cell homogenates are $5 \%$ of the samples used for IP assays. In c, data are expressed as mean values \pm S.D.; $n=3$ independent experiments. Statistical significance was determined by one-way ANOVA followed by Dunnett's test for multiple comparison, ${ }^{*} P<0.05 ;{ }^{* *} P<0.01$ 
a
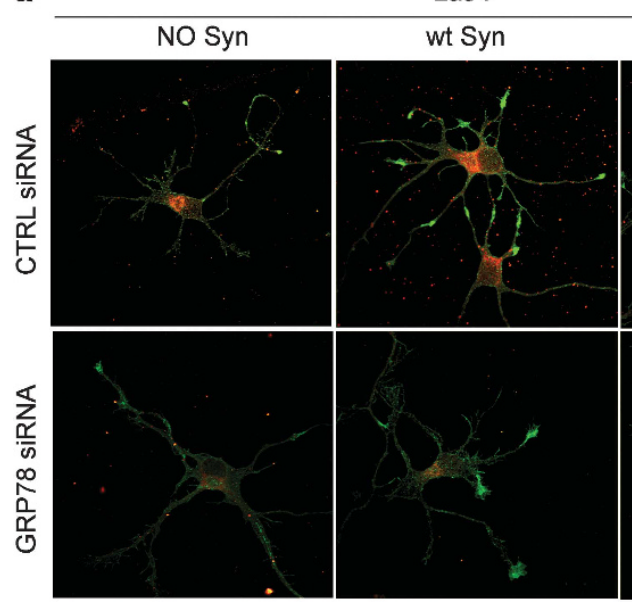

c

Syn

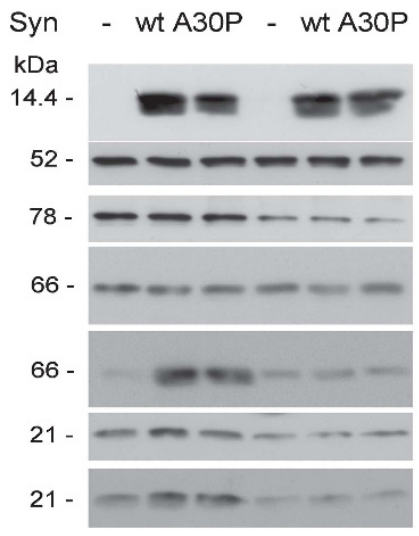

Syn

ß-tubulin II

GRP78

PAK 2

p-PAK 2

cofilin 1

p-cofilin 1
LatA

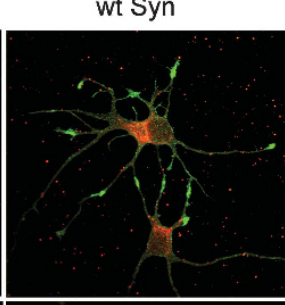

A30P Syn

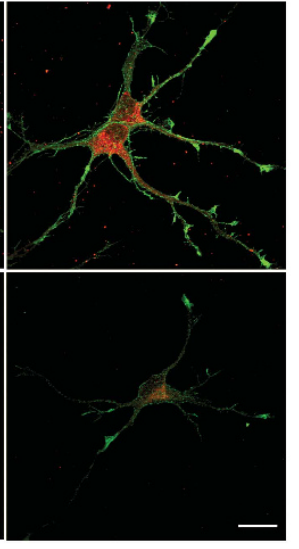

d

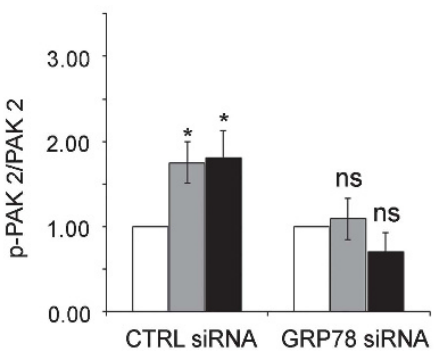

b

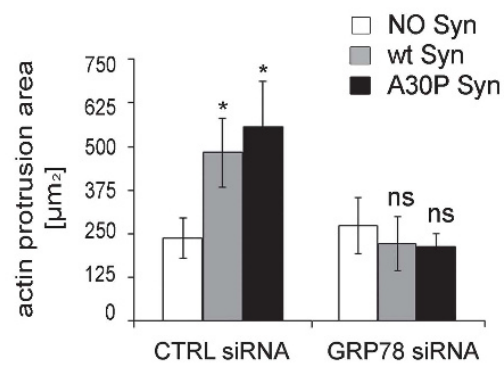

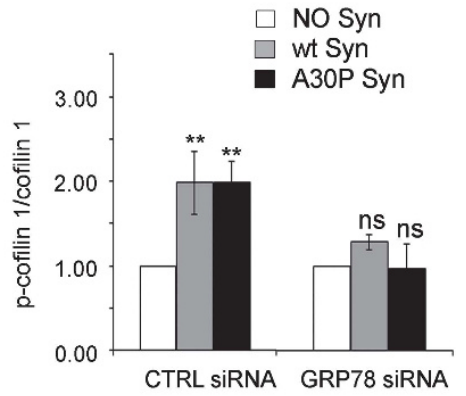

Figure 6 GRP78 downregulation prevents the effects triggered by extracellular wt and A30P Syn. (a) Fluorescence analysis of embryonic hippocampal neurons electroporated at 1 DIV with GRP78 siRNA sequences or with not-targeting siRNA sequences (CTRL siRNA), incubated at 4 DIV for $1 \mathrm{~h}$ in the absence or presence of $1 \mu \mathrm{M}$ purified wt or A30P Syn and then treated for $1 \mathrm{~h}$ with $1 \mu \mathrm{M}$ LatA. Neurons were processed for fluorescent phalloidin staining (F-actin, green) and indirect immunofluorescence of GRP78 (red). (b) Quantitative evaluation of actin protrusion areas in neurons treated as in a. Actin protrusion areas were calculated by subtracting $\beta$-tubulin III projection areas from F-actin projection areas. (c) Representative immunoblots of cell homogenates from hippocampal neurons electroporated at 1 DIV with GRP78 siRNA sequences and incubated at 4 DIV in the absence or presence of purified wt or A30P Syn as in a. $\beta$-Tubulin III is shown as an internal standard. (d) Quantification of the ratio between p-PAK 2 and total PAK 2 (left panel) and p-cofilin 1 and total cofilin 1 (right panel) bands, analyzed by densitometry, for each experimental condition in c; values are normalized to those of control samples not incubated with Syn (NO Syn). In $\mathbf{b}$ and $\mathbf{d}$, data are expressed as mean values \pm S.D. In $\mathbf{b}, n=60$ neurons from three independent cultures; in d, $n=3$ independent experiments. Statistical significance was determined by one-way ANOVA followed by Dunnett's test for multiple comparison, ${ }^{*} P<0.05 ;{ }^{* *} P<0.01$; $\mathrm{ns}=$ not significant. Bar in $\mathbf{a}: 20 \mu \mathrm{m}$

\section{The actin cytoskeleton is a target for Syn in primary} fibroblasts from PD patients. To verify whether an excess of Syn could affect actin cytoskeleton also in cells from PD patients, we used primary fibroblasts, which are known to express Syn. Fibroblasts isolated from human skin biopsies of four patients bearing genetic PD (3 Syn-duplication; 1 Syntriplication) appeared larger than control fibroblasts and showed a dramatic increase in the number and thickness of actin stress fibers (Figures $7 a$ and $b$ ). As expected, PD fibroblasts expressed increased amounts of Syn as compared with controls (Figure 7c). The increased expression of Syn correlated with a threefold increase in the phosphocofilin 1/cofilin 1 ratio in PD fibroblasts with respect to controls (Figures $7 \mathrm{c}$ and d, Supplementary Figure 6). Thus, in PD fibroblasts, Syn activates the same pathway that leads to actin stabilization in neurons, resulting in increased formation of stress fibers.
To address whether the increased Syn expression leads to its increased release in the medium, $48 \mathrm{~h}$-conditioned media were immunoprecipitated with anti-Syn antibody (Figure 7e). Syn was present at concentrations of 2.5 and $1 \mathrm{pM}$ in the medium of Syn $3 \mathrm{X}$ and control fibroblasts, respectively.

To demonstrate that Syn, released in excess from PD fibroblasts, is the cause of the increased formation of stress fibers, we compared the actin morphology of control fibroblasts incubated with either $0.1 \mu \mathrm{M}$ purified Syn or the medium derived from Syn $3 X$ fibroblasts; as control, fibroblasts were incubated with the medium derived from distinct control cells. In the presence of extracellular Syn, the actin cytoskeleton appeared altered (Figure 7f), similarly to that of PD fibroblasts (Figure 7a). Importantly, immunodepletion of Syn from Syn 3X fibroblasts medium was no longer inducing formation of actin stress fibers (Figures $7 f$ and $g$ ). 


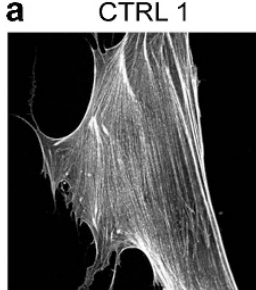

Syn $2 X 1$
CTRL 2

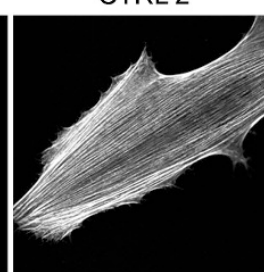

Syn 2 X 2
CTRL 3

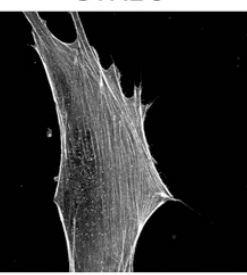

Syn $2 \times 3$
CTRL 4

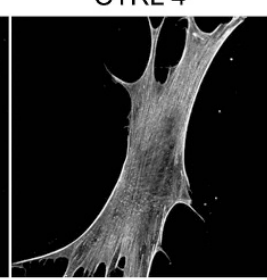

Syn $3 X$
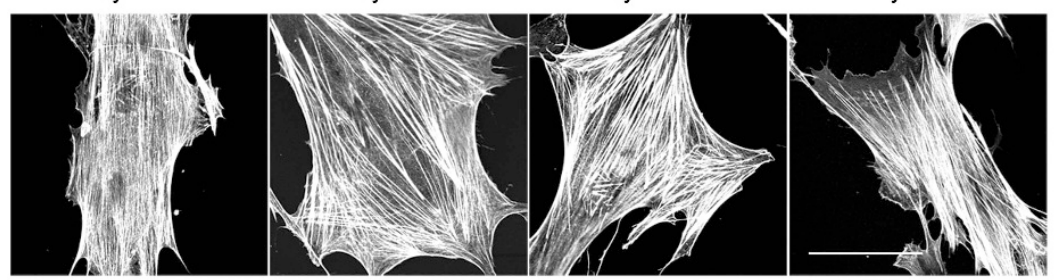

c

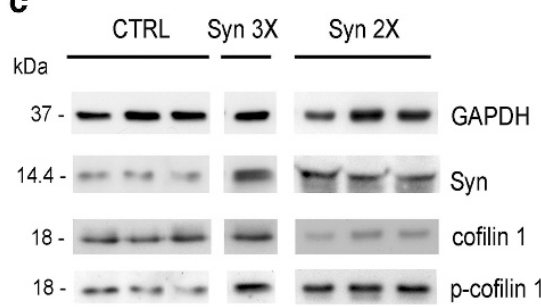

d

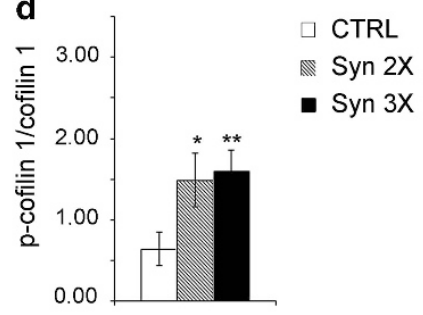

b

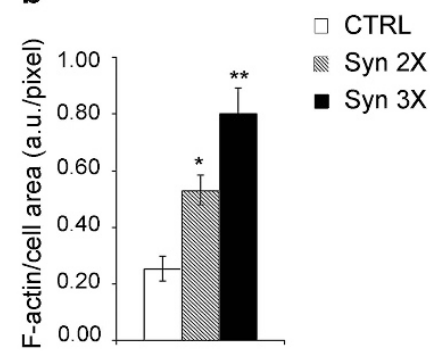

e

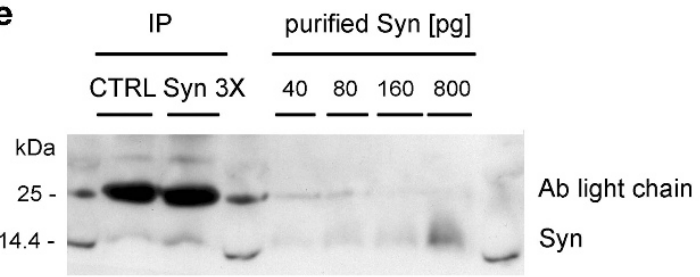

f

+ CTRL
medium

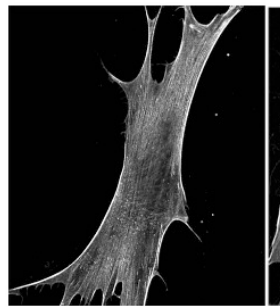

+ wt Syn

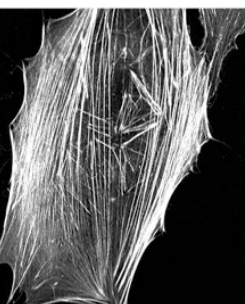

+ Syn $3 X$ medium

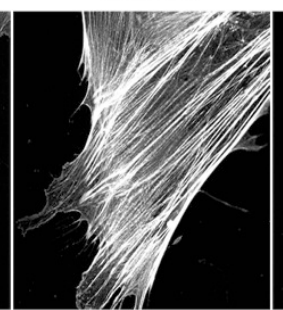

+ Syn $3 X$ cleared medium

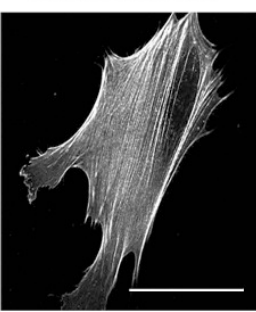

g

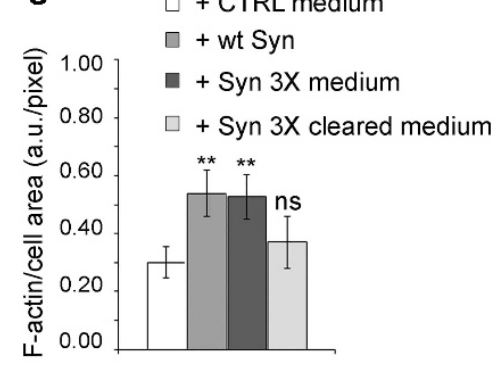

Figure 7 Actin cytoskeleton displays alterations in fibroblasts from PD-affected patients carrying Syn multiplications. (a) Fluorescent phalloidin staining (F-actin) of human skin fibroblasts, dissected from four controls (CTRL 1-4) and four disease-affected patients carrying Syn duplication (Syn 2X1-3) or triplication (Syn 3X). (b) Quantification of F-actin staining in fibroblasts as in a. For each image, F-actin fluorescent staining is normalized to the cell area. Values in the bar graph represent the average of four CTRL, three Syn 2X and Syn 3X fibroblasts. (c) Immunoblots of lysates from human skin fibroblasts dissected from CTRL subjects or PD-affected patients (Syn 2X, Syn 3X). The lysates from the various patients were run on different gels, but the nitrocellulose membranes were processed in parallel. GAPDH is shown as an internal standard. (d) Quantification of the ratio between p-cofilin 1 and total cofilin 1 bands, analyzed by densitometry, in CTRL, Syn $2 X$ or Syn 3X fibroblasts. (e) IP assay with anti-Syn antibody from the media of CTRL or Syn 3X fibroblasts, collected after $48 \mathrm{~h}$ in culture. Increasing concentrations of purified Syn (40-800 pg) were loaded in parallel and used to calculate the amount of released Syn, precipitated from the media. (f) Fluorescent phalloidin staining (F-actin) of control fibroblasts cultured ON in the presence of the medium derived from distinct control fibroblasts ( + CTRL medium), of $0.1 \mu \mathrm{M}$ purified wt Syn ( + wt Syn), of the medium derived from Syn 3X fibroblasts ( + Syn 3X medium) or of the medium from Syn $3 X$ fibroblasts cleared by anti-Syn antibody ( + Syn $3 X$ cleared medium). (g) Quantitative evaluation of F-actin staining per cell area in fibroblasts as in $\mathbf{f}$, and calculated as in $\mathbf{b}$. In $\mathbf{b}$ and $\mathbf{g}$, data are expressed as mean values \pm S.D.; $n=60$ fibroblasts from three independent experiments. In $\mathbf{d}$, data are expressed as mean values \pm S.D.; $n=3$ independent experiments. Statistical significance was determined by one-way ANOVA followed by Dunnett's test for multiple comparison, ${ }^{\star} P<0.05$; ${ }^{* *} P<0.01$. Bars in a and $\mathrm{f:} 30 \mu \mathrm{m}$ 
These data provide evidence that disease-affected fibroblasts, carrying multiplications of Syn-coding gene, show specific alterations in their actin cytoskeleton organization, which depend on the presence of Syn in the extracellular milieu.

\section{Discussion}

The present results reinforce the hypothesis that release of Syn is a physiological event, which also contributes to the spreading of Syn-derived pathology, ${ }^{26}$ indicating extracellular Syn as a biomarker for related diseases. ${ }^{27}$

We previously demonstrated that actin is a target for intracellular Syn, and that wt and A30P-mutated Syn differentially modulate actin dynamics. ${ }^{13}$ Here, we provide evidences that the extracellular accumulation of either wt or A30P Syn correlates with an unbalance of actin turnover, resulting in microfilament stabilization. Extracellular Syns, delivered either as purified monomeric proteins in unfolded conformation or with the medium of overexpressing neurons, induced the formation of large lamellipodia-like actin protrusions along the neurites and at their tips. Microfilaments appeared stabilized, with depolymerization-resistant actin structures clustered in spots along the cell periphery. Functional consequences, such as defects in axon elongation, are present at early stages of neuronal development.

Albeit exosomes might be involved in Syn release, ${ }^{22}$ the culture media collected from electroporated neurons contained soluble, rather than membrane-associated Syns. Syns could be extracellularly released in picomolar concentration, in agreement with the circulating levels of the protein detected in biological fluids. ${ }^{15}$ The evidence that high concentrations of extracellularly applied wt Syn $(1 \mu \mathrm{M})$ were acting similarly to A30P mutant (active at lower dosage, that is, $0.1 \mu \mathrm{M}$ ) is consistent with the pathological role acquired by the wt protein upon accumulation, as it occurs in PD cases of gene multiplications. $^{5-7}$ The different concentration of purified or released Syns needed to achieve a similar effect on actin cytoskeleton might be due to conformational changes of their structure. During their extracellular release, Syns are exposed to the acidic environment of intracellular organelles, which might influence their conformation, ${ }^{28}$ whereas it is possible that only a small fraction of extracellularly added recombinant Syns are active.

In our in vitro system, the exposure to extracellular Syns did not result in neuronal toxicity, and cell loss was evident only after 14 days of exposure. This suggests that actin alterations due to monomeric Syns may represent an early event in Syninduced pathology, taking place before toxicity via non-cell autonomous pathways, and opening the possibility of their use as early biomarkers of the disease.

We report that extracellular Syns tune the activation of modulators of actin dynamics. In particular, the presence of Syns correlated with increased activation of the small GTPase Rac 1, which is known to promote actin polymerization at the leading edge of cells. ${ }^{29}$ Activation of Rac 1 resulted in increased phosphorylation of its main substrate PAK 2, leading to phosphorylation of cofilin 1, an essential regulator of actin dynamics, and to inhibition of its actin depolymerizing activity. ${ }^{30}$ Inactivation of cofilin 1 is consistent with the observed morphological changes of neuronal cytoskeleton, that is, actin stabilization and resistance to depolymerization. ${ }^{31}$

We identified GRP78 as the protein that, in the presence of extracellular Syns, relocates to the plasma membrane and interacts with Syns. GRP78 is a major ER chaperone protein ${ }^{23}$ involved in the UPR, which is frequently altered in neurodegenerative diseases, ${ }^{32}$ known to interact with Syn in the ER in stress conditions. ${ }^{33,34}$ We provided evidence that the binding of extracellular Syns to GRP78 occurs at the cell surface and leads to its clustering in discrete membrane domains, possibly as a consequence of inhibition of its lateral movement along the plasma membrane, and of its decreased recycling. Alternatively, Syn might induce clustering of GRP78 at the cell surface through changes in membrane composition, curvature and integrity. ${ }^{35}$ Indeed, alteration of membrane processes has been proposed as the mechanism leading to surface-accumulation of transferrin receptor by extracellular Syn oligomers. ${ }^{36}$

Experiments of siRNA-mediated knockdown of GRP78 indicated that GRP78 is necessary for the activation of the signaling cascade leading to inactivation of cofilin 1 and cytoskeleton alterations, possibly through the formation of macromolecular complexes that act as signaling platforms. We can hypothesize that the decrease in the expression levels of total cofilin 1 observed upon downregulation of GRP78 might be due to a negative feedback activated by the neurons to counterbalance hyperactive, unphosphorylated cofilin 1.

The relevance of cytoskeletal alterations in the pathogenesis of PD was confirmed by the analysis of primary fibroblasts from patients with early onset genetic $P D$, due to duplication or triplication of SNCA. Increased Syn level was shown also in fibroblasts from sporadic PD patients, confirming the relevance of Syn dosage in pathology. ${ }^{37}$ Syn multiplications correlated with a dramatic increase in randomly oriented actin stress fibers, consistent with previous observations on fibroblasts from idiopathic PD patients. ${ }^{38}$ Diseased cells also showed an increase in the phosphorylation of cofilin 1, similarly to neurons exposed to Syns. The increase in Syn expression in PD fibroblasts correlated with an increased release of Syn that was responsible for propagation of the observed cytoskeletal alterations; in fact, the medium derived from Syn 3X fibroblasts triggered the pathological actin phenotype in control cells, and lost this capability once cleared of Syn.

In conclusion we identified a new mechanism and a novel target for extracellular Syn at the plasma membrane and demonstrated that the impaired dynamics of the cytoskeleton in neurons is due to interaction of monomeric Syn with surface-exposed GRP78. The model shown in Figure 8 illustrates the hypothetical mechanism of action of endogenous and exogenous Syn. Although intracellular Syn contributes to maintain actin in a depolymerized state through direct binding and sequestration of monomers, high concentrations of extracellular Syn activate a pathway leading to cofilin 1 inactivation and stabilization of microfilaments, thereby contrasting the physiological regulatory role of the endogenous protein.

Our results also suggest that primary fibroblasts could represent a useful tool for screening and early diagnosis. 


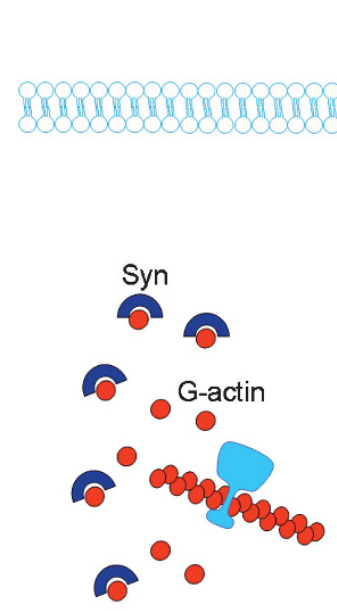

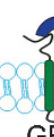
(n)

syn

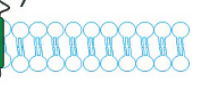

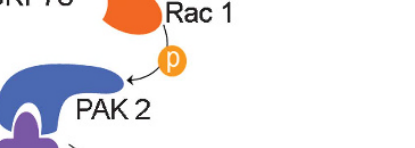

Figure 8 Hypothetical model of Syn mechanisms of action. Wt intracellular Syn sequester actin monomer in a contest in which the severing protein cofilin 1 is active. Increased levels of extracellular Syn shift the balance of actin dynamics toward polymerization. This effect is achieved through the inactivation of cofilin 1 by phosphorylation, triggered by the clustering of GRP78 at the plasma membrane and activation of the depicted signaling pathway

The microtubular cytoskeleton has already been identified as a target for therapy in neurodegenerative diseases; ${ }^{39,40}$ here, we propose that drugs acting on the actin cytoskeleton have potential use in the treatment of PD. In addition, as depletion of the medium from Syn was sufficient to reduce the propagation of cytoskeletal pathology, the reduction of the circulating levels of Syn might be an additional therapeutic target at early stage of the disease, as suggested and reviewed in. ${ }^{41}$

\section{Materials and methods}

Reagents. All solvents were from VWR (Radnor, PA, USA) and the other chemicals were from Merck (Darmstadt, Germany).

Antibodies and fluorescent probes. Mouse monoclonal antibodies: antiactivated caspase 3 and anti-cofilin 1 (Abcam, Cambridge, MA, USA); anti-Rac 1 and anti-Syn (BD Transduction Laboratories, Franklin Lakes, NJ, USA); anti $\beta$-tubulin III (Covance, Emervylle, CA, USA).

Rabbit polyclonal antibodies: anti-phosphorylated cofilin 1 and anti-PAK 1,2 (Santa Cruz Biotechnology, Santa Cruz, CA, USA); anti-GRP94 (Abcam); antiGAPDH, anti-phosphorylated elF2 $\alpha$ and anti-phosphorylated PAK 2 (Cell Signaling, Danvers, MA, USA); anti-GFP (Molecular Probes-Life Technologies, Carlsbad, CA, USA); anti-GRP78 (StressMarq, Victoria, BC, Canada); anti- $\beta$-tubulin III (Covance).

Fluorescent-conjugated secondary antibodies, either coupled to fluorescein isothiocyanate (FITC), tetramethylrhodamine isothiocyanate or pacific blue were from Jackson ImmunoResearch (Newmarket Suffolk, UK); horseradish peroxidaseconjugated secondary antibodies were from Bio-Rad (Hercules, CA, USA); FITCconjugated phalloidin, HOECHST, sytox orange and 5,5',6,6'-tetrachloro$1,1^{\prime}, 3,3^{\prime}$ tetraethilbenzimidazolocarbocyanine iodide (JC-1) were from Molecular Probes-Life Technologies.

Plasmids and siRNA-targeted sequences. The DNA fragments coding for human wt or A30P Syn including the stop codon were inserted into pEGFP-N1 vector (Clontech, Saint-Germain-en-Laye, France) and used for electroporation experiments in hippocampal neurons; as a control, the empty pEGFP-N1 vector was used. The GST-PAK-CRIB plasmid was a gift from Professor Ivan de Curtis (Ospedale San Raffaele and Vita-Salute University, Milan, Italy). Sequences for
siRNA siGENOME were obtained from Dharmacon-Thermo Scientific (Lafayette, CO, USA). The siRNA sequence targeting mouse GRP78 was 5'-GGAAUGACCC UUCGGUGCA-3' (accession number NM_022310, catalog number D-040337-02); the catalog number for the non-targeting siRNA was D-001206-14. MISSION esiRNA sequences were obtained from Sigma-Aldrich (St. Louis, MO, USA). The catalog number of esiRNA sequences targeting mouse GRP78 was EMU051691 (Ensembl accession number ENSMUSG00000026864, NCBI accession number NP_071705), whereas MISSION esiRNA targeting Firefly Luciferase was used as a negative control (catalog number EHUFLUC).

Protein purification. Constructs encoding the human full-length wt or A30P Syn inserted in the pET21d plasmid were a kind gift from Dr. Brett Lauring (Columbia University, New York, NY, USA). Purification was performed as in, ${ }^{13}$ followed by gel filtration in Superdex 75 10/300 column (GE Healthcare Life Sciences, Uppsala, Sweden). Denaturing of Syns was achieved by boiling the proteins for $5 \mathrm{~min}$ at $100^{\circ} \mathrm{C}$. Syn peptides (amino acids 12-23, 34-45 and 61-78) were obtained from Primmbiotech (Milano, Italy).

Primary embryonic mouse cultures. Primary neuronal cultures were prepared from the hippocampi or cortices dissected from C57BL/6 S E18 mice carrying a chromosomal deletion encompassing the locus gene for Syn (Harlan, Udine, Italy). ${ }^{42}$ Animals were maintained in a pathogen-free animal facility. All experiments were performed in strict accordance with experimental procedures approved by the Animal Care and Use Committee of the Ospedale San Raffaele (Milan, Italy) and by the Italian Ministry of Health.

Primary hippocampal neurons were prepared $\mathrm{as}^{43}$ and plated either on glass coverslips (density of $2500 \mathrm{cell} / \mathrm{s} / \mathrm{cm}^{2}$ ), or on plastic petri dishes (density of 10000 cells $/ \mathrm{cm}^{2}$ ) (see Supplementary Materials and Methods, Hippocampal neuronal cultures). Cortical neurons were prepared as in ${ }^{44}$ and plated on plastic Petri dishes (density $26000 \mathrm{cells} / \mathrm{cm}^{2}$ ) (see Supplementary Materials and Methods).

Primary skin fibroblast cultures. Human fibroblasts (see Supplementary Materials and Methods) were cultured in Eagle's minimal essential medium (MEM, Gibco-Life Technologies, Carlsbad, CA, USA) supplemented with $10 \%$ fetal bovine serum (EuroClone GE Healthcare Life Sciences), $110 \mathrm{mg} / \mathrm{l}$ sodium pyruvate (Sigma-Aldrich), $2 \mathrm{mM}$ glutamine, $100 \mathrm{U} / \mathrm{ml}$ penicillin and $100 \mu \mathrm{g} / \mathrm{ml}$ streptomycin (all from Gibco-Life Technologies) and maintained at $37^{\circ} \mathrm{C}$ in a humidified atmosphere with $5 \% \mathrm{CO}_{2}$. In all assays, the fibroblast passage numbers were matched $(<10)$.

Electroporation of hippocampal neurons. Primary hippocampal neurons were electroporated in suspension immediately after dissociation, ${ }^{45}$ using the Basic Nucleofector Kit for primary neurons on the 0-05 program (Amaxa Biosystems, Cologne, Germany). Neurons were electroporated with the constructs of interest ( $3 \mu \mathrm{g}$ DNA plasmid/1500000 neurons) or with the specific siRNAtargeted sequences (with a mixture containing $(2 \mu \mathrm{g}$ esiRNA $+2 \mathrm{ng}$ siGENOME siRNA)/1500 000 neurons) and plated on glass coverslips, or on plastic Petri dishes, as described above. Electroporated neurons were used at 4 DIV.

Exosome preparation. Neuronal culture media were collected after 4 DIV and centrifuged at $4000 \times g$ for $10 \mathrm{~min}$ at $4{ }^{\circ} \mathrm{C}$ to remove cell debris. The isolation of the externalized membrane vesicles was performed as previously described. ${ }^{22}$ Briefly, the culture medium was centrifuged at $100000 \times g$ for $2 \mathrm{~h}$ at $4{ }^{\circ} \mathrm{C}$ in a TLA Beckman Coulter ultracentrifuge (Palo Alto, CA, USA). The supernatant (S100) was collected and concentrated by a speed vacuum concentrator (Eppendorf concentrator plus, Hamburg, Germany) and the pellet (P100) containing externalized vesicles was reconstituted in Laemmli buffer. S100 and P100 fractions were then analyzed by SDS-PAGE and immunoblotting (see Supplementary Materials and Methods).

Remodeling of the actin cytoskeleton and image analyses. Hippocampal neurons were grown in culture for 1, 4 or 14 DIV and incubated at $37^{\circ} \mathrm{C}$ in the presence or absence of wt or A30P Syn $(1 \mathrm{~h}$ with $1 \mu \mathrm{M}$ purified recombinant wt or A30P Syn; overnight treatment with Syn-containing media); control incubations were obtained by adding the chromatographic elution buffer (for neurons which were treated with purified Syns), or by applying the medium collected from cultures of pEGFP-N1-electroporated neurons (for neurons that were treated with Syn-containing media). Neurons were fixed at rest or after LatA (Molecular Probes-Life Technologies)-induced-actin depolymerization ${ }^{17,46}$ and 
then processed for whole-cell indirect immunofluorescence (see Materials and Methods); treatment with LatA was performed for $1 \mathrm{~h}$ at $37^{\circ} \mathrm{C}$ at $1 \mu \mathrm{M}$ final concentration. Images were acquired in an Axiovert 135 inverted microscope (Zeiss, Oborkochen, Germany), equipped with epifluorescence optics and recorded with a C4742 ORCA II cooled charge-coupled service camera (Hamamatsu Photonics, Hamamatsu City, Japan). Confocal images were acquired in a Leica SP2 confocal microscope, using a Leica 63X objective lens (NA 1.4; Leica Microsystems, Wetzlar, Germany). Actin protrusion areas were calculated by subtracting the projection areas of $\beta$-tubulin III staining from the projection areas of F-actin staining (fluorescent phalloidin). All images were analyzed with the custommade ImageJ routine (NIH, Bethesda, MD, USA), after subtraction of the same arbitrary threshold. The development and organization of neural processes were evaluated using Sholl analysis. ${ }^{47}$ Concentric rings with a regular radial increment of $7 \mu \mathrm{m}$ were centered on the neuronal soma (considered as having a $20 \mu \mathrm{m}$ diameter) and the number of processes intersecting each of the five rings was counted. The length of each axon was measured using an ImageJ plugin.

Rac 1 activity assay. Hippocampal neurons were grown in culture for 14 DIV and then incubated for $1 \mathrm{~h}$ in the absence or presence of $1 \mu \mathrm{M}$ wt or A30P Syn. Neurons were homogenized at $4{ }^{\circ} \mathrm{C}$ in extraction buffer $(10 \%$ glycerol, $100 \mathrm{mM}$

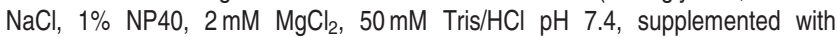
protease and phosphatase inhibitors (Sigma-Aldrich)) and centrifuged at $10000 \times g$ for $10 \mathrm{~min}$ at $4{ }^{\circ} \mathrm{C}$. Samples $(150 \mu$ g protein) were incubated for $1 \mathrm{~h}$ at $4^{\circ} \mathrm{C}$ with $50 \mu$ l glutathione-agarose beads (Sigma-Aldrich), pre-adsorbed with the bacterially purified GST-PAK-CRIB fusion protein, as described in. ${ }^{48}$ As a positive control, a fraction (1/10) of the neuronal lysate was loaded in vitro for

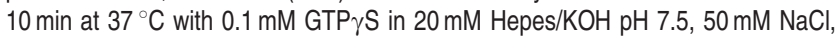
$1 \mathrm{mM}$ EDTA and $1 \mathrm{mM}$ dithiotreitol, as in Albertinazzi et al. ${ }^{49}$ Loading was stopped by adding $\mathrm{MgCl}_{2}$ to $5 \mathrm{mM}$ final concentration. After performing the Rac1-GTP pull-down assays, samples were analyzed by SDS-PAGE and immunoblotting (see Supplementary Materials and Methods).

Cell-surface labeling and IP assays. Hippocampal neurons were grown in culture for 14 DIV and then incubated for 1 or $4 \mathrm{~h}$ in the presence or absence of $1 \mu \mathrm{M}$ wt or A30P Syn, washed twice with cold Hank's Balanced Buffered Saline containing $1 \mathrm{mg} / \mathrm{ml}$ glucose and incubated with the impermeable biotin-conjugated compound EZ-Link Sulfo-NHS-LC-Biotin (Amersham-GE Healthcare) at $0.6 \mathrm{mg} / \mathrm{ml}$ final concentration. ${ }^{50}$ The biotinylation reaction was stopped by incubation with $1 \%$ bovine serum albumin (BSA, Sigma-Aldrich) for $10 \mathrm{~min}$ at $4{ }^{\circ} \mathrm{C}$ and neurons were lysed in $1 \%$ SDS, $50 \mathrm{mM}$ Hepes/KOH pH 7.4, $150 \mathrm{mM} \mathrm{NaCl}, 5 \mathrm{mM}$ EDTA pH 8.0, supplemented with protease and phosphatase inhibitors. SDS-solubilised proteins were isolated by spinning at $300000 \times g$ in a TLA 100 Beckman Coulter ultracentrifuge and then diluted $1: 10$ to reach a $0.1 \%$ SDS final concentration. Solubilised proteins were used to perform pull-down assay of biotin-labeled proteins, or IP assays with a specific antibody. Biotin-labeled proteins were precipitated by pull-down assay with $50 \mu \mathrm{l}$ streptavidin-conjugated sepharose beads (Amersham-GE Healthcare); for IP assays of GRP78, biotinylated protein samples were incubated with $2 \mu \mathrm{g}$ anti-GRP78 antibody adsorbed to $50 \mu$ l protein G-sepharose beads (Amersham-GE Healtcare). Beads were incubated for $2 \mathrm{~h}$ at $4{ }^{\circ} \mathrm{C}$ on a rotating wheel, washed three times in $0.1 \%$ SDS, $50 \mathrm{mM}$ Hepes $/ \mathrm{KOH}$ $\mathrm{pH} 7.4,150 \mathrm{mM} \mathrm{NaCl}, 5 \mathrm{mM}$ EDTA pH 8.0 and protein samples were eluted in Laemmli buffer and analyzed by SDS-PAGE and immunoblotting (see Supplementary Materials and Methods).

Atomic force microscopy. Cortical neurons, grown in culture for $10 \mathrm{DIV}$, were incubated with $5 \mu \mathrm{M}$ wt Syn for $2 \mathrm{~h}$ at $37^{\circ} \mathrm{C}$, fixed with $4 \%$ paraformaldehyde and then incubated for $1 \mathrm{~h}$ with anti-Syn (1:50) or anti-GRP78 (1:20) antibodies. Neurons were then fixed again and stored at $4^{\circ} \mathrm{C}$ until AFM acquisition.

Functionalized colloidal probes (see Supplementary Material and Methods) were mounted on a Nanowizard III system (JPK Instruments, Berlin, Germany) coupled to an Axio Observer D1 (Zeiss) inverted optical microscope. A single force spectroscopy point was selected on the cell soma directly from the optical image; the exact superimposition between optical images and the AFM tip position was obtained by using the Direct Overlay routine of the AFM acquisition software (JPK Instruments). A maximum force of $0.6 \mathrm{nN}$ was applied to the cell and the bead/cell contact was maintained for $3 \mathrm{sec}$. The velocity of the probe retraction was $3 \mu \mathrm{m} / \mathrm{sec}$. The same AFM system was used also with the NPG functional probes. Force maps of $60 \times 60$ force spectroscopy points were collected on $3 \times 3 \mu \mathrm{m}^{2}$ area on the cell soma. In the case of the NPG probes, the maximum force applied was reduced to
$0.25 \mathrm{nN}$, in order to avoid significant indentation. The retraction velocity was kept constant at $3 \mu \mathrm{m} / \mathrm{s}$ and the tip/cell contact was maintained for $0.1 \mathrm{sec}$. Negative controls were obtained by adding a secondary antibody solution $(0.02 \mathrm{mg} / \mathrm{ml})$ in the AFM measurement chambers, to block the recognition signal. Thirty minutes after, the excess secondary antibody was removed by perfusing new buffer in the AFM liquid chamber and the recognition measurement was repeated on the same cell, taking into account a small drift due to thermal effect. The actual spring constant of each cantilever was determined in situ, using the thermal noise method. ${ }^{51}$

Image elaboration and statistics. Image composition and drawing were done with the use of Adobe Photoshop (Adobe System, San Jose, CA, USA). Data were analyzed using Microsoft Excel software and expressed as mean values \pm standard deviation (S.D.). Prism software (Graph Pad, La Jolla, CA, USA) was used to perform one-way ANOVA followed by Dunnetts's test for multiple comparisons, to assess the statistical significance ( $P$-values $<0.05$ were considered significant).

For AFM experiments, F-D curves were analyzed using the JPK Data Processing software or by homemade Matlab routines (Natick, MA, USA). F-D curves were corrected for the bending of the cantilever ${ }^{52}$ to calculate the tip-sample separation (F-I curves). To quantify the adhesion between cell and the colloidal probes, the total area delimited by the part of the retracted curve below the F-D curve baseline, providing an estimate of the detachment work ( $W_{\text {detach }}$ ), was considered. F-I curves acquired with NPG probes were fitted with the worm-like chain model in order to eliminate the unspecific unbinding events. The number of rupture events was considered, as well as the maximum rupture force $\left(F_{\max }\right)$ for each curve. The number of rupture events and the $F_{\max }$ of each curve were reported in bi-dimensional maps. The same maps were acquired after blocking the probe recognition capability, as described in the previous section.

\section{Conflict of Interest}

The authors declare no conflict of interest.

Acknowledgements. We thank Brett Lauring (Columbia University, New York) for Syn plasmids and Ivan de Curtis (San Raffaele Scientific Institute, Milan) for the GST-PAK-CRIB plasmid. We thank Stefano Goldwurm for providing control and Syn $2 X$ fibroblasts; the samples were obtained from the 'Cell Line and DNA Biobank from Patients affected by Genetic Diseases' (G. Gaslini Institute, Genoa, Italy) and 'Parkinson Institute Biobank' (Milan, Italy; http:/www.parkinsonbiobank. com/) of the Telethon Network Genetic Biobank (http://www.biobanknetwork.org, project No. GTB07001). Syn 3X fibroblasts were from the NINDS Repository (ccr.coriell.org/ninds; sample number ND27760). Part of this work was carried out in ALEMBIC, an advanced microscopy laboratory established by the San Raffaele Scientific Institute and the Vita-Salute San Raffaele University. This work was supported by Telethon Foundation GGP10109 (to EC).

\section{Author Contributions}

SB, EC, FV, CC conceived and designed the experiments; SB, AM, GR, HT, ST performed the experiments; SB, AM, CC analyzed the data and; SB, EC, FV wrote the paper.

1. Maroteaux L, Campanelli JT, Scheller RH. Synuclein: a neuron-specific protein localized to the nucleus and presynaptic nerve terminal. J Neurosci 1988; 8: 2804-2815.

2. Vekrellis K, Xilouri M, Emmanouilidou E, Rideout HJ, Stefanis L. Pathological roles of alpha-synuclein in neurological disorders. Lancet Neurol 2011; 10: 1015-1025.

3. Spillantini MG, Schmidt ML, Lee VM, Trojanowski JQ, Jakes R, Goedert M. Alpha-synuclein in Lewy bodies. Nature 1997; 388: 839-840.

4. Houlden $\mathrm{H}$, Singleton $A B$. The genetics and neuropathology of Parkinson's disease. Acta Neuropathol 2012; 124: 325-338.

5. Chartier-Harlin MC, Kachergus J, Roumier C, Mouroux V, Douay X, Lincoln S et al. Alpha-synuclein locus duplication as a cause of familial Parkinson's disease. Lancet 2004; 364: 1167-1169.

6. Singleton $A B$, Farrer $M$, Johnson $J$, Singleton $A$, Hague $S$, Kachergus $\mathrm{J}$ et al. alpha-Synuclein locus triplication causes Parkinson's disease. Science 2003; 302: 841.

7. Ibanez P, Bonnet AM, Debarges B, Lohmann E, Tison F, Pollak P et al. Causal relation between alpha-synuclein gene duplication and familial Parkinson's disease. Lancet 2004; 364: 1169-1171.

8. Venda LL, Cragg SJ, Buchman VL, Wade-Martins R. alpha-Synuclein and dopamine at the crossroads of Parkinson's disease. Trends Neurosci 2010; 33: 559-568. 
9. Fortin DL, Troyer MD, Nakamura K, Kubo S, Anthony MD, Edwards RH. Lipid rafts mediate the synaptic localization of alpha-synuclein. J Neurosci 2004; 24: 6715-6723.

10. Auluck PK, Caraveo G, Lindquist S. alpha-Synuclein: membrane interactions and toxicity in Parkinson's disease. Annu Rev Cell Dev Biol 2010; 26: 211-233.

11. Lucking CB, Alpha-synuclein Brice A. and Parkinson's disease. Cell Mol Life Sci 2000; 57 1894-1908

12. Lykkebo S, Jensen $\mathrm{PH}$. Alpha-synuclein and presynaptic function: implications for Parkinson's disease. Neuromol Med 2002; 2: 115-129.

13. Sousa VL, Bellani S, Giannandrea M, Yousuf M, Valtorta F, Meldolesi J et al. \{alpha\}-synuclein and Its A30P mutant affect actin cytoskeletal structure and dynamics. Mol Biol Cell 2009; 20: 3725-3739.

14. Cingolani LA, Goda Y. Actin in action: the interplay between the actin cytoskeleton and synaptic efficacy. Nat Rev Neurosci 2008; 9: 344-356.

15. Marques O, Outeiro TF. Alpha-synuclein: from secretion to dysfunction and death. Cell Death Dis 2012; 3: e350.

16. Dunning CJ, Reyes JF, Steiner JA, Brundin P. Can Parkinson's disease pathology be propagated from one neuron to another? Prog Neurobiol 2012; 97: 205-219.

17. Spector I, Braet F, Shochet NR, Bubb MR. New anti-actin drugs in the study of the organization and function of the actin cytoskeleton. Microsc Res Tech 1999; 47: 18-37.

18. Bartels T, Choi JG, Selkoe DJ. alpha-Synuclein occurs physiologically as a helically folded tetramer that resists aggregation. Nature 2011; 477: 107-110.

19. Burre J, Vivona S, Diao J, Sharma M, Brunger AT, Sudhof TC. Properties of native brain alpha-synuclein. Nature 2013; 498: E4-E6; discussion E6-E7.

20. Crowet JM, Lins L, Dupiereux I, Elmoualija B, Lorin A, Charloteaux B et al. Tilted properties of the 67-78 fragment of alpha-synuclein are responsible for membrane destabilization and neurotoxicity. Proteins 2007; 68: 936-947.

21. Fantini J, Carlus D, Yahi N. The fusogenic tilted peptide (67-78) of alpha-synuclein is a cholesterol binding domain. Biochim Biophys Acta 2011; 1808: 2343-2351.

22. Emmanouilidou E, Melachroinou K, Roumeliotis T, Garbis SD, Ntzouni M, Margaritis LH et al. Cell-produced alpha-synuclein is secreted in a calcium-dependent manner by exosomes and impacts neuronal survival. J Neurosci 2010; 30: 6838-6851.

23. Ni M, Zhang Y, Lee AS. Beyond the endoplasmic reticulum: atypical GRP78 in cell viability, signalling and therapeutic targeting. Biochem J 2011; 434: 181-188.

24. Ludwig M, Dettmann W, Gaub HE. Atomic force microscope imaging contrast based on molecular recognition. Biophys J 1997; 72: 445-448.

25. Dupres V, Verbelen C, Dufrene YF. Probing molecular recognition sites on biosurfaces using AFM. Biomaterials 2007; 28: 2393-2402.

26. Lee SJ. Origins and effects of extracellular alpha-synuclein: implications in Parkinson's disease. J Mol Neurosci 2008; 34: 17-22.

27. Parnetti L, Castrioto A, Chiasserini D, Persichetti E, Tambasco N, El-Agnaf $O$ et al. Cerebrospinal fluid biomarkers in Parkinson disease. Nat Rev Neurol 2013; 9 ; $131-140$

28. Ahn KJ, Paik SR, Chung KC, Kim J. Amino acid sequence motifs and mechanistic features of the membrane translocation of alpha-synuclein. J Neurochem 2006; 97 265-279.

29. Nobes CD, Hall A. Rho, rac, and cdc42 GTPases regulate the assembly of multimolecular focal complexes associated with actin stress fibers, lamellipodia, and filopodia. Cell 1995; 81: $53-62$.

30. Sarmiere PD, Bamburg JR. Regulation of the neuronal actin cytoskeleton by ADF/cofilin. J Neurobiol 2004; 58: 103-117.

31. Chai X, Forster E, Zhao S, Bock HH, Frotscher M. Reelin stabilizes the actin cytoskeleton of neuronal processes by inducing n-cofilin phosphorylation at serine3. J Neurosci 2009 ; 29: 288-299.
32. Hoozemans JJ, van Haastert ES, Eikelenboom P, de Vos RA, Rozemuller JM, Scheper W. Activation of the unfolded protein response in Parkinson's disease. Biochem Biophys Res Commun 2007; 354: 707-711.

33. Colla E, Coune P, Liu Y, Pletnikova O, Troncoso JC, Iwatsubo T et al. Endoplasmic reticulum stress is important for the manifestations of alpha-synucleinopathy in vivo. J Neurosci 2012; 32: 3306-3320.

34. Colla E, Jensen PH, Pletnikova O, Troncoso JC, Glabe C, Lee MK. Accumulation of toxic alpha-synuclein oligomer within endoplasmic reticulum occurs in alpha-synucleinopathy in vivo. J Neurosci 2012; 32: 3301-3305.

35. Westphal $\mathrm{CH}$, Chandra SS. Monomeric synucleins generate membrane curvature. J Biol Chem 2013; 288: 1829-1840.

36. Chai YJ, Kim D, Park J, Zhao H, Lee SJ, Chang S. The secreted oligomeric form of alpha-synuclein affects multiple steps of membrane trafficking. FEBS Lett 2013; 587: 452-459.

37. Hoepken HH, Gispert S, Azizov M, Klinkenberg M, Ricciardi F, Kurz A et al. Parkinson patient fibroblasts show increased alpha-synuclein expression. Exp Neurol 2008; 212 307-313

38. Cartelli D, Goldwurm S, Casagrande F, Pezzoli G, Cappelletti G. Microtubule destabilization is shared by genetic and idiopathic Parkinson's disease patient fibroblasts. PLOS ONE 2012; 7: e37467.

39. Cartelli D, Casagrande F, Busceti CL, Bucci D, Molinaro G, Traficante A et al. Microtubule alterations occur early in experimental parkinsonism and the microtubule stabilizer epothilone D is neuroprotective. Sci Reports 2013; 3: 1837.

40. Zhang B, Carroll J, Trojanowski JQ, Yao Y, Iba M, Potuzak JS et al. The microtubulestabilizing agent, epothilone $D$, reduces axonal dysfunction, neurotoxicity, cognitive deficits, and Alzheimer-like pathology in an interventional study with aged tau transgenic mice. J Neurosci 2012; 32: 3601-3611

41. Danzer KM, McLean PJ. Drug targets from genetics: alpha-synuclein. CNS Neurol Disord Drug Targets 2011; 10: 712-723

42. Specht CG, Schoepfer R. Deletion of the alpha-synuclein locus in a subpopulation of C57BL/6 J inbred mice. BMC Neurosci 2001; 2: 11

43. Banker GA, Cowan WM. Rat hippocampal neurons in dispersed cell culture. Brain Res 1977; 126: 397-342.

44. Brewer GJ. Isolation and culture of adult rat hippocampal neurons. J Neurosci Methods 1997; 71: 143-155

45. Gartner A, Collin L, Lalli G. Nucleofection of primary neurons. Methods Enzymol 2006; 406: 374-388.

46. Coue M, Brenner SL, Spector I, Korn ED. Inhibition of actin polymerization by latrunculin A. FEBS Lett 1987; 213: 316-318.

47. Sholl DA. Dendritic organization in the neurons of the visual and motor cortices of the cat. J Anat 1953; 87: 387-406.

48. Sander EE, van Delft S, ten Klooster JP, Reid T, van der Kammen RA, Michiels F et al Matrix-dependent Tiam1/Rac signaling in epithelial cells promotes either cell-cell adhesion or cell migration and is regulated by phosphatidylinositol 3-kinase. J Cell Biol 1998; 143: 1385-1398.

49. Albertinazzi C, Za L, Paris S, de Curtis I. ADP-ribosylation factor 6 and a functional PIX/ p95-APP1 complex are required for Rac1B-mediated neurite outgrowth. Mol Biol Cell 2003; 14: 1295-1307.

50. Daniels GM, Amara SG. Selective labeling of neurotransmitter transporters at the cell surface. Methods Enzymol 1998; 296: 307-318.

51. Hutter JL, Bechhoefer J. Calibration of atomic-force microscope tips. Rev Sci Instrum 1993; 64: $1868-1873$.

52. Azeloglu EU, Costa KD. Atomic force microscopy in mechanobiology: measuring microelastic heterogeneity of living cells. Methods Mol Biol 2011; 736: 303-329.

Supplementary Information accompanies this paper on Cell Death and Differentiation website (http://www.nature.com/cdd) 